\title{
A higher order perfectly matched layer formulation for finite-difference time-domain seismic wave modeling
}

\author{
David P. Connolly ${ }^{1}$, Antonios Giannopoulos ${ }^{2}$, and Michael C. Forde ${ }^{2}$
}

\begin{abstract}
We have developed a higher order perfectly matched layer (PML) formulation to improve the absorption performance for finite-difference time-domain seismic modeling. First, we outlined a new unsplit "correction" approach, which allowed for traditional, first-order PMLs to be added directly to existing codes in a straightforward manner. Then, using this framework, we constructed a PML formulation that can be used to construct higher order PMLs of arbitrary order. The greater number of degrees of freedom associated with the higher order PML allow for enhanced flexibility of the PML stretching functions, thus potentially facilitating enhanced absorption performance. We found that the new approach can offer increased elastodynamic
\end{abstract}

absorption, particularly for evanescent waves. We also discovered that the extra degrees of freedom associated with the higher order PML required careful optimization if enhanced absorption was to be achieved. Furthermore, these extra degrees of freedom increased the computational requirements in comparison with first-order schemes. We reached our formulations using one compact equation thus increasing the ease of implementation. Additionally, the formulations are based on a recursive integration approach that reduce PML memory requirements, and do not require special consideration for corner regions. We tested the new formulations to determine their ability to absorb body waves and surface waves. We also tested standard staggered grid stencils and rotated staggered grid stencils.

\section{INTRODUCTION}

The finite-difference time-domain (FDTD) modeling techniques are commonly used to simulate elastodynamic wave propagation for the purposes of seismic exploration. Absorbing boundary conditions $(\mathrm{ABCs})$ are typically placed at domain edges to prevent boundary reflections from contaminating results. The ABC's performance dictates how far it can be placed from the modeling areas of concern without causing reflections. Therefore, a highly effective $\mathrm{ABC}$ can be placed in close proximity to the modeling area of interest, thus significantly reducing the computational workload.

Damping zones (Cerjan et al., 1985), continued fraction conditions (Guddati and Lim, 2006), optimized boundary conditions (Peng and Toksoz, 1995), viscous boundaries (Lysmer and Kuhlemeyer, 1969; Kouroussis et al., 2011), paraxial conditions (Higdon, 1986), and nonlocal operators (Hagstrom and Hariharan, 1998) approaches have been attempted to absorb outgoing seismic waves. Although these techniques generally performed well for waves arriving perpendicular to the boundary, their performance was reduced for waves impinging at low angles of incidence. This is undesirable for $2 \mathrm{D} / 3 \mathrm{D}$ seismic wave modeling because the wave patterns are composed of large variations in the incidence angle.

Berenger (1994, 1996) introduces a "perfectly matched layer" (PML) technique to absorb electromagnetic waves (i.e., Maxwell's equations) using a series of finite layers, each with identical material properties to the modeling domain, to gradually damp outgoing waves. It offered high performance and was capable of absorbing waves independent of the arrival angle. Chew and Weedon (1994) quickly extend this PML formulation to include a stretching of real and imaginary spatial coordinates, thus offering the potential for additional absorption.

Using a similar implementation to the electromagnetic wave approach, Chew and Lui (1996) adapt the PML condition to offer absorption for seismic waves. Since then, several approaches have

\footnotetext{
Manuscript received by the Editor 13 April 2014; revised manuscript received 11 August 2014; published online 1 December 2014.

${ }^{1}$ Heriot-Watt University, Institute for Infrastructure \& Environment, Edinburgh, UK. E-mail: d.connolly@hw.ac.uk.

${ }^{2}$ University of Edinburgh, School of Engineering, Edinburgh, UK. E-mail: a.giannopoulos@ed.ac.uk; m.forde@ed.ac.uk.

(C) 2014 Society of Exploration Geophysicists. All rights reserved.
} 
been used to implement PML conditions for elastodynamic problems (Hastings et al., 1996; Basu and Chopra, 2004; Basu, 2009). One drawback of such approaches was that reflections were encountered at grazing incidences. This was because although the PML reflection coefficient was theoretically zero before discretization, after discretization it was not zero. Analytical expressions were used to overcome this for the electromagnetic case; however, due to the increased complexity of the underlying elastodynamic equations, this was more challenging (Collino and Tsogka, 2001).

An alternative approach for electromagnetics was to modify the complex coordinate stretching function within the PML (Kuzuoglu and Mittra, 1996; Roden and Gedney, 2000). This C-PML, or complex frequency shifted (CFS)-PML, was a more attractive option for the elastodynamics case and therefore was further explored by Festa and Vilotte (2005). The theory behind this method was that it would offer much improved absorption for waves propagating at low grazing angles (i.e., for long distances within the PML region). The CFS-PML proved popular and has since been developed for poroelastic (Martin et al., 2008) and anisotropic media (Bécache et al., 2003).

Early CFS-PML conditions were implemented using an artificial splitting of velocity and stress fields, in a similar manner to some of the early non-CFS-PML conditions including Chew and Weedon (1994). This splitting procedure made PML implementation in traditional FDTD codes challenging because two different sets of equations were required for each PML and non-PML region. In addition, such implementations were not well posed mathematically (Abarbanel and Gottlieb, 1997).

To avoid field splitting, convolution terms (Komatitsch and Martin, 2007), auxiliary differential equations (ADEs) (Martin et al., 2010), and integration approaches (Drossaert and Giannopoulos, 2007) were also investigated. Because convolution is generally regarded as computationally inefficient, recent focus has shifted to $\mathrm{ADE}$ and integral term implementations.

Martin et al. (2010) outline a nonconvolutional ADE PML approach in which a fourth-order Runge-Kutta scheme is used in conjunction with eighth-order Holberg space discretization. This formulation was shown to have high accuracy and to be stable for up to 100,000 time steps. Additionally, Martin et al. (2010) investigate the potential to extend this ADE-PML condition to higher order PMLs but conclude that no significant performance benefit was capable.

Zhang and Shen (2010) built on the work of Martin et al. (2010) and outline a similar ADE-PML fourth-order Runge-Kutta scheme that resulted in a complete set of first-order differential equations. This meant that the same FDTD implementation could be used to solve the ADE C-PML equations and the interior-domain equations.

An alternative approach to avoid field splitting is outlined by Drossaert and Giannopoulos (2007) through the use of recursive integration (RI)-PML. This technique used an extended trapezoidal rule to integrate the PML time derivatives thus negating the requirement to split fields or use an ADE formulation. The RI-PML approach required an equal amount of memory in comparison with split-field formulations and slightly less memory than the ADE approach.

One of the shortcomings of using the new CFS-PML was that although it offered improved absorption for low-incident waves, the application of the underlying filter reduced the absorption performance of the PML for waves arriving at high incidences (i.e., at $90^{\circ}$ angles).
In an attempt to maximize absorption for low- and high-incidence waves for electromagnetic wave modeling, Correia and Jin (2005) propose higher order PML formulations. These formulations used a combination of non-CFS and CFS stretching functions. They find that enhanced performance was achievable because the non-CFS component aided in the absorption of high-incident waves whereas the CFS component aided the absorption of the low-incident waves.

This paper extends the PML implementation described by Giannopoulos (2012) to the seismic wave equation using an RI-PML approach. It does so based on a new and improved "correction PML" implementation approach, which is also outlined. The higher order PML has the potential to utilize a greater number of degrees of freedom in comparison with the traditional first-order PML condition, thus offering greater absorption. Improved absorption is desirable because it allows for reduced domain sizes, which is particularly important for 3D problems (Laghrouche and Le Houédec, 1994; Kouroussis et al., 2014). This potential for increased absorption is highlighted through several comparisons with alternative first-order PML conditions. It should be noted that although the new PML is termed "higher order," this has no bearing on its compatibility with space and time discretization.

\section{IMPLEMENTING PML THROUGH A CORRECTION TECHNIQUE}

To derive the new time-domain correction PML, it was first necessary to work in the frequency domain before transferring back to the time domain and then using the trapezoidal rule to solve the resulting integral. Therefore, using a stretched coordinate system, the $2 \mathrm{D}$ (3D formulation shown in Appendix A) frequency-domain elastodynamic velocity-stress equations (based on the original timedomain equations as outlined by Virieux, 1986; Graves, 1996) took the form

$$
\begin{gathered}
j \omega \tilde{v}_{x}=b\left(\frac{1}{s_{x}} \frac{\partial \tilde{\sigma}_{x x}}{\partial x}+\frac{1}{s_{z}} \frac{\partial \tilde{\sigma}_{x z}}{\partial z}\right), \\
j \omega \tilde{v}_{z}=b\left(\frac{1}{s_{x}} \frac{\partial \tilde{\sigma}_{x z}}{\partial x}+\frac{1}{s_{z}} \frac{\partial \tilde{\sigma}_{z z}}{\partial z}\right), \\
j \omega \tilde{\sigma}_{x x}=(\lambda+2 \mu) \frac{1}{s_{x}} \frac{\partial \tilde{v}_{x}}{\partial x}+\lambda \frac{1}{s_{z}} \frac{\partial \tilde{v}_{z}}{\partial z}, \\
j \omega \tilde{\sigma}_{z z}=(\lambda+2 \mu) \frac{1}{s_{z}} \frac{\partial \tilde{v}_{z}}{\partial z}+\lambda \frac{1}{s_{x}} \frac{\partial \tilde{v}_{x}}{\partial x},
\end{gathered}
$$

and

$$
j \omega \tilde{\sigma}_{x z}=\mu\left(\frac{1}{s_{x}} \frac{\partial \tilde{v}_{z}}{\partial x}+\frac{1}{s_{z}} \frac{\partial \tilde{v}_{x}}{\partial z}\right),
$$

where the frequency-domain velocity and stress components are denoted by $\tilde{v}$ and $\tilde{\sigma}$, respectively; $\omega$ represents circular frequency; $\lambda$ and $\mu$ are the lamés coefficients; and $b$ is the buoyancy. The coordinate axes are defined by $x$ and $z$, and $s_{x}$ and $s_{z}$ are the PML 
stretching functions (in the $x$ - and $z$-directions, respectively), which served to absorb outgoing waves:

$$
s_{u_{i}}=\kappa_{u_{i}}+\frac{d_{u_{i}}}{\alpha_{u_{i}}+j \omega} .
$$

In equation $6, d_{u_{i}}, \kappa_{u_{i}}$, and $\alpha_{u_{i}}$ are the attenuation coefficients used to describe the loss within the PML region. The subscript $u$ is used to denote the staggered grid component, e.g., stress/velocity, and the subscript $i$ is used to describe the direction of attenuation $(u \in[u, v]$ and $i \in[x, z]$ ). Additionally, the variable transform $j=\sqrt{-1}$ and $\psi_{u}$ are defined as

$$
\psi_{u}=\frac{1-s_{u}}{s_{u}}=\frac{1}{s_{u}}\left(\frac{1-s_{u}}{1}\right)=\frac{1}{s_{u}}-1
$$

or

$$
\frac{1}{s_{u}}=\left(1+\psi_{u}\right)
$$

Rearranging equations $1-5$ in terms of $\psi_{u}$ gives

$$
\begin{gathered}
j \omega \tilde{v}_{x}=b\left(\left(1+\psi_{x}\right) \frac{\partial \tilde{\sigma}_{x x}}{\partial x}+\left(1+\psi_{z}\right) \frac{\partial \tilde{\sigma}_{x z}}{\partial z}\right), \\
j \omega \tilde{v}_{z}=b\left(\left(1+\psi_{x}\right) \frac{\partial \tilde{\sigma}_{x z}}{\partial x}+\left(1+\psi_{z}\right) \frac{\partial \tilde{\sigma}_{z z}}{\partial z}\right), \\
j \omega \tilde{\sigma}_{x x}=(\lambda+2 \mu)\left(1+\psi_{x}\right) \frac{\partial \tilde{v}_{x}}{\partial x}+\lambda\left(1+\psi_{z}\right) \frac{\partial \tilde{v}_{z}}{\partial z}, \\
j \omega \tilde{\sigma}_{z z}=(\lambda+2 \mu)\left(1+\psi_{z}\right) \frac{\partial \tilde{v}_{z}}{\partial z}+\lambda\left(1+\psi_{x}\right) \frac{\partial \tilde{v}_{x}}{\partial x},
\end{gathered}
$$

and

$$
j \omega \tilde{\sigma}_{x z}=\mu\left(\left(1+\psi_{x}\right) \frac{\partial \tilde{v}_{z}}{\partial x}+\left(1+\psi_{z}\right) \frac{\partial \tilde{v}_{x}}{\partial z}\right) .
$$

A comparison between equations 1-5 and 9-13 reveals that the stretched velocity/stress equations were analogous to an addition of field-dependent variables. To display this with greater clarity, equations $9-13$ are rearranged and rewritten as

$$
\begin{aligned}
& j \omega \tilde{v}_{x}=b\left(\frac{\partial \tilde{\sigma}_{x x}}{\partial x}+\frac{\partial \tilde{\sigma}_{x z}}{\partial z}\right)+b\left(\tilde{J}_{x x}+\tilde{J}_{x z}\right), \\
& j \omega \tilde{v}_{z}=\tilde{b}\left(\frac{\partial \tilde{\sigma}_{x z}}{\partial x}+\frac{\partial \tilde{\sigma}_{z z}}{\partial z}\right)+b\left(\tilde{J}_{x z}+\tilde{J}_{z z}\right),
\end{aligned}
$$

$$
j \omega \tilde{\sigma}_{x x}=(\lambda+2 \mu) \frac{\partial \tilde{v}_{x}}{\partial x}+\lambda \frac{\partial \tilde{v}_{z}}{\partial z}+\left((\lambda+2 \mu) \tilde{M}_{x x}+\lambda \tilde{M}_{x z}\right),
$$

$$
j \omega \tilde{\sigma}_{z z}=(\lambda+2 \mu) \frac{\partial \tilde{v}_{z}}{\partial z}+\lambda \frac{\partial \tilde{v}_{x}}{\partial x}+\left((\lambda+2 \mu) \tilde{M}_{z z}+\lambda \tilde{M}_{z x}\right),
$$

and

$$
j \omega \tilde{\sigma}_{x z}=\mu\left(\frac{\partial \tilde{v}_{z}}{\partial x}+\frac{\partial \tilde{v}_{x}}{\partial z}\right)+\mu\left(\tilde{M}_{z x}+\tilde{M}_{x z}\right),
$$

where $\tilde{J}$ is a field-dependent variable used to correct the velocity components and $\tilde{M}$ is a field-dependent variable used to correct the stress components. Then $\tilde{J}$ and $\tilde{M}$ are given by

$$
\tilde{J}_{x u}=\psi_{u} \frac{\partial \tilde{\sigma}_{x u}}{\partial u}
$$

and

$$
\tilde{M}_{x u}=\psi_{u} \frac{\partial \tilde{v}_{v}}{\partial u}
$$

with $u, v \in\{x, z\}$ and $u \neq v$.

From equations $14-18$, it is clear that the velocity and stress values of the stretched coordinates in the PML region (i.e., where $\psi_{u} \neq 0$ ) could be calculated through an addition of equations 19 and 20 to the previously calculated velocity and stress values. Thus, for an existing FDTD script, the field-dependent variables could be added to the regions in which absorption was required.

To do so, first, the original update equations are computed as if there is no PML present (i.e., rigid boundary condition), using arbitrary time-stepping techniques such as those described in Virieux (1986) and Graves (1996). Then, the cells within the PML regions are updated by adding the correction terms to the original values as previously calculated. This can be seen as "correcting" the original update terms to account for the presence of the PML. In comparison, for traditional noncorrection PML schemes such as those by Komatitsch and Martin (2007), the stress and velocity values are calculated at the same instance for the interior domain and the PML regions. This can impact the ease of implementation.

Therefore, the key benefits of the new first-order correction PML are as follows:

1) When adding PML to an existing code, no revisions to the original code need to be made.

2) PML corner regions do not require any special consideration.

3) Programming complexity is significantly reduced.

It should be noted that equations 14-18 were cast in the frequency domain. To calculate the equivalent time-domain equations, recursive integration is required. The following section describes this process for the development of a higher order PML, using this correction approach. Therefore, the resulting equations needed to 
calculate the first-order time-domain correction terms are described by equations $62-66$.

\section{DEVELOPMENT OF A HIGHER ORDER PML}

The new correction PML (equations 14-18) facilitated straightforward implementation of the PML stretching functions because its formulation did not require any modification of the underlying FDTD update scheme. This made it well suited to provide a foundation for implementing arbitrary stretching functions. Therefore, it was used to provide an efficient and straightforward PML formulation for $N$ th-order stretching.

\section{Stretching function definitions}

For the 2D case, six correction terms were required to describe the attenuation of all velocity and stress field variables (three $J_{u u}$ for velocity terms and three $M_{u u}$ for stress terms). Despite this, for brevity, only the derivation of $J_{x z}$ (i.e., the correction term required to partly describe the stretching of the velocity components) is outlined. All other correction terms $\left(J_{u u}\right.$ and $\left.M_{u u}\right)$ can be found analogously.

First, equations 7 and 19 were combined, which leads to

$$
\tilde{J}_{x u}=\left(\frac{1}{s_{u}}-1\right) \frac{\partial \tilde{\sigma}_{x u}}{\partial u} .
$$

Then, considering only the vertical components (i.e., $u=z$ ) and rearranging resulted in

$$
\frac{\partial \tilde{\sigma}_{x z}}{\partial z}=s_{z}\left(\tilde{J}_{x z}+\frac{\partial \tilde{\sigma}_{x z}}{\partial z}\right) .
$$

Additionally, for the $N$ th order, stretching the overall stretching function is defined as

$$
s_{u}=\prod_{i=1}^{N} s_{u_{i}}
$$

This means that the overall stretching function is the product of all other stretching functions from 1 to $N$. When combined with equation 22 , this yields

$$
\frac{\partial \tilde{\sigma}_{x z}}{\partial z}=\left(\prod_{i=1}^{N} s_{z_{i}}\right)\left(\tilde{J}_{x z}+\frac{\partial \tilde{\sigma}_{x z}}{\partial z}\right),
$$

where $N \in[\Re]$, meaning that potentially the combination of an infinite number of stretching functions could be calculated. Despite this, it is possible to define the stretching functions needed to describe the overall stretching function, using only three sets of $i$ :

1) one stretching function for the first-order stretch $(i=1)$

2) one stretching function for the final-order stretch $(i=N)$

3 ) one stretching function for all the stretching functions between the first and last $(1<i<N)$
To calculate these three stretching functions, a set of functions $\Psi_{x z_{i}}$ is defined for $i \in[1, N-1]$ as

$$
\Psi_{x z_{i}}=\left(\prod_{m=i+1}^{N} s_{z_{m}}\right)\left(\tilde{J}_{x z}+\frac{\partial \tilde{\sigma}_{x z}}{\partial z}\right) .
$$

Using equations 24 and 25 to eliminate $\tilde{J}_{x z}$ leads to

$$
\frac{\partial \tilde{\sigma}_{x z}}{\partial z} / \Psi_{x z_{i}}=s_{z_{i}} .
$$

Therefore, the first-order stretching function $(i=1)$ is equivalent to

$$
\Psi_{x z_{1}}=\frac{1}{s_{z_{1}}} \frac{\partial \tilde{\sigma}_{x z}}{\partial z} .
$$

Then, combining equations 22 and 27 allows for the calculation of equation 28. This is used to describe the stretching function between the first and the final stretching function $(i \in[2, N-1])$ :

$$
\Psi_{x z_{i}}=\frac{1}{s_{z_{i}}} \Psi_{i-1}
$$

Last, combining equations 22 and 28 results in the final stretching function $(i=N)$ :

$$
\left(\tilde{J}_{x z}+\frac{\partial \tilde{\sigma}_{x z}}{\partial z}\right)=\frac{1}{s_{z_{N}}} \Psi_{x z_{N-1}} .
$$

\section{Domain transformation}

The stretching functions 27-29 are defined using frequency-domain terms. To implement them within a time-domain finite-difference model, they have to be reformulated in the time domain.

To facilitate this transformation, first, stretching function 6 is substituted into 26, giving

$$
\kappa_{z_{1}} \Psi_{x z_{1}}+\frac{d_{z_{1}}}{\alpha_{z_{1}}+i \omega} \Psi_{x z_{1}}=\frac{\partial \tilde{\sigma}_{x z}}{\partial z} .
$$

With the intention of solving for $\Psi_{x z_{1}}$, both sides are multiplied by $\left(\alpha_{z_{1}}+i \omega\right)$ as

$$
\left(\alpha_{z_{1}} \kappa_{z_{1}}+d_{z_{1}}\right) \Psi_{x z_{1}}+i \omega \kappa_{z_{1}} \Psi_{x z_{1}}=\alpha_{z_{1}} \frac{\partial \tilde{\sigma}_{x z}}{\partial z}+i \omega \frac{\partial \tilde{\sigma}_{x z}}{\partial z}
$$

To prime equation 31 for transformation, it is rearranged and similar terms are grouped together as

$$
\Psi_{x z_{1}}=\frac{1}{\kappa_{z_{1}}} \frac{\partial \tilde{\sigma}_{x z}}{\partial z}+\frac{1}{i \omega}\left[\frac{\alpha_{z_{1}}}{\kappa_{z_{1}}} \frac{\partial \tilde{\sigma}_{x z}}{\partial z}-\frac{\left(\alpha_{z_{1}} \kappa_{z_{1}}+d_{z_{1}}\right)}{\kappa_{z_{1}}} \Psi_{x z_{1}}\right] \text {. }
$$

The relationship $\frac{1}{i \omega} \tilde{A}(W)=\int_{0}^{t} A(t) \delta t$ is then used to make the transform trivial. The post transformation stretching function is equivalent to the time domain integral: 


$$
\Psi_{x z_{1}}=\frac{1}{\kappa_{z_{1}}} \frac{\partial \sigma_{x z}}{\partial z}+\int_{0}^{t} \frac{\alpha_{z_{1}}}{\kappa_{z_{1}}} \frac{\partial \sigma_{x z}}{\partial z}-\frac{\left(\alpha_{z_{1}} \kappa_{z_{1}}+d_{z_{1}}\right)}{\kappa_{z_{1}}} \Psi_{x z_{1}} \delta t .
$$

\section{Application of the extended trapezoidal rule}

The time integral (equation 32) is not yet ready to be implemented within an FDTD scheme. For PML applications, the high-accuracy and low-memory requirements associated with using the trapezoidal integration rule (Drossaert and Giannopoulos, 2007) have made it an attractive choice. Therefore, this is the approach taken.

The higher order PML can be implemented in any staggered grid FDTD formulation. For the purpose of this derivation, its implementation was assumed to be within a velocity-stress grid that was staggered in space and time. Therefore, if " $t$ " is time, when $t=n$, the velocity components were first calculated, and then, the PML regions are updated.

It should be noted that all field quantities are assumed to be zero for $t \leq 0$. Also, the index notation $J_{a 1}^{a 2}$ is used, where $a 1$ defines the index for spatial discretization and $a 2$ denotes the index for time discretization. Consequently, the application of the extended trapezoidal rule results in

$$
\begin{aligned}
\Psi_{x z_{1}}^{n+\frac{1}{2}} & =\frac{1}{\kappa_{z_{1}}} \frac{\partial \sigma_{x z}^{n+\frac{1}{2}}}{\partial \mathrm{z}} \\
& +\sum_{p=0}^{n=1}\left[\frac{\alpha_{z 1} \Delta t}{\kappa_{z_{1}}} \frac{\partial \sigma_{x z}^{p+\frac{1}{2}}}{\partial z}-\frac{\left(\alpha_{z 1} \kappa_{z_{1}}+d_{z_{1}}\right) \Delta t}{\kappa_{z_{1}}} \Psi_{x z_{1}}^{p+\frac{1}{2}}\right] \\
& +\frac{\Delta t}{2} \frac{\alpha_{z 1}}{\kappa_{z_{1}}} \frac{\partial \sigma_{x z}^{n+1 / 2}}{\partial z}-\frac{\Delta t}{2} \frac{\left(\alpha_{z 1} \kappa_{z_{1}}+d_{z_{1}}\right)}{\kappa_{z_{1}}} \Psi_{x z_{1}}^{n+1 / 2} .
\end{aligned}
$$

This is rearranged and then solved for $\Psi_{x z_{1}}^{n+1 / 2}$, giving

$$
\begin{aligned}
\Psi_{x z_{1}}^{n+1 / 2} & =\frac{2+\Delta t \alpha_{z 1}}{2 \kappa_{z 1}+\Delta t\left(\alpha_{z 1} \kappa_{z_{1}}+d_{z_{1}}\right)} \frac{\partial \sigma_{x z}^{n+1 / 2}}{\partial z} \\
& +\frac{2 \kappa_{z 1}}{2 \kappa_{z 1}+\Delta t\left(\alpha_{z 1} \kappa_{z_{1}}+d_{z_{1}}\right)} \sum_{p=0}^{n=1}\left[\frac{\alpha_{z 1} \Delta t}{\kappa_{z_{1}}} \frac{\partial \sigma_{x z}^{p+1 / 2}}{\partial z}\right. \\
& \left.-\frac{\left(\alpha_{z 1} \kappa_{z_{1}}+d_{z_{1}}\right) \Delta t}{\kappa_{z_{1}}} \Psi_{x z_{1}}^{p+1 / 2}\right] .
\end{aligned}
$$

The summation term on the right side is then replaced by $\Phi_{x z_{1}}^{n-1 / 2}$ as

$$
\Phi_{x z_{1}}=\sum_{p=0}^{n-1}\left[\frac{\alpha_{z_{1}} \Delta t}{\kappa_{z_{1}}} \frac{\partial \sigma_{x z}^{p+1 / 2}}{\partial z}-\frac{\left(\alpha_{z_{1}} \kappa_{z_{1}}+d_{z_{1}}\right) \Delta t}{\kappa_{z_{1}}} \Psi_{x z_{1}}^{p+1 / 2}\right]
$$

Physically, this holds the approximation to the integral at the previous time step. Computationally, this variable is updated after the stresses, velocities, and their corresponding correction terms, but at the same time instance. Therefore,

$$
\begin{aligned}
\Psi_{x z_{1}}^{n+1 / 2} & =\frac{2+\Delta t \alpha_{z 1}}{2 \kappa_{z_{1}}+\Delta t\left(\alpha_{z 1} \kappa_{z_{1}}+d_{z_{1}}\right)} \frac{\partial \sigma_{x z}^{n+1 / 2}}{\partial z} \\
& +\frac{2 \kappa_{z_{1}}}{2 \kappa_{z_{1}}+\Delta t\left(\alpha_{z 1} \kappa_{z_{1}}+d_{z_{1}}\right)} \Phi_{x z_{1}}^{n-1 / 2}
\end{aligned}
$$

where $\Phi_{x z_{1}}^{n+1 / 2}$ is defined as

$$
\Phi_{x z_{1}}^{n+1 / 2}=\Phi_{x z_{1}}^{n-1 / 2}+\frac{\alpha_{z 1} \Delta t}{\kappa_{z 1}} \frac{\sigma_{x z}^{n+1 / 2}}{\partial z}-\frac{\Delta t\left(\alpha_{z 1} \kappa_{z 1}+d_{z 1}\right)}{\kappa_{z 1}} \Psi_{x z_{1}}^{n+1 / 2}
$$

\section{Calculating the $N$ th-order correction terms}

The next step toward deriving an expression for $J_{x z}^{n+1 / 2}$ is to eliminate $\Psi_{x z_{1}}^{n+1 / 2}$ from the update of $\Phi_{x z_{1}}^{n+1 / 2}$. This is done using equations 35 and 38 and results in

$$
\begin{aligned}
\Phi_{x z_{1}}^{n+1 / 2} & =\frac{2 \kappa_{z 1}-\Delta t\left(\alpha_{z 1} \kappa_{z 1}+d_{z 1}\right)}{2 \kappa_{z 1}+\Delta t\left(\alpha_{z 1} \kappa_{z 1}+d_{z 1}\right)} \Phi_{x z_{1}}^{n+1 / 2} \\
& -\frac{2 d_{z 1} \Delta t}{\left(2 \kappa_{z 1}+\Delta t\left(\alpha_{z 1} \kappa_{z 1}+d_{z 1}\right)\right) \kappa_{z 1}} \frac{\partial \sigma_{x z}^{n+1 / 2}}{\partial z} .
\end{aligned}
$$

Upon inspection of equation 39, it is found that for $i \in[2, N-1]$, $\Psi_{x z_{i}}$ could be calculated in an analogous manner to $\Psi_{x z_{1}}$, which results in

$$
\begin{aligned}
\Psi_{x z_{i}}^{n+1 / 2} & =\frac{2+\Delta t \alpha_{z i}}{2 \kappa_{z i}+\Delta t\left(\alpha_{z i} \kappa_{z i}+d_{z i}\right)} \Psi_{x z_{i-1}}^{n+1 / 2} \\
& +\frac{2 \kappa_{z i}}{2 \kappa_{z i}+\Delta t\left(\alpha_{z i} \kappa_{z i}+d_{z i}\right)} \Phi_{x z_{i}}^{n-1 / 2}
\end{aligned}
$$

Correspondingly, the previous time integrals $\Phi_{x z_{i}}$ for $i \in[2, N]$ are updated as

$$
\begin{aligned}
\Phi_{x z_{i}}^{n+1 / 2} & =\frac{2 \kappa_{z i}+\Delta t\left(\alpha_{z i} \kappa_{z i}+d_{z i}\right)}{2 \kappa_{u i}+\Delta t\left(\alpha_{z i} \kappa_{z i}+d_{z i}\right)} \Phi_{x z_{i}}^{n-1 / 2} \\
& -\frac{2 \sigma_{z i} \Delta t}{\left(2 \kappa_{z i}+\Delta t\left(\alpha_{z i} \kappa_{z i}+d_{z i}\right)\right) \kappa_{z i}} \Psi_{x z_{i-1}}^{n+1 / 2} .
\end{aligned}
$$

Last, using the same methodology as that to arrive at equation 40 , equations 29 and 40 are used to create an overall formulation for $J_{x z}^{n+1 / 2}$ as

$$
\begin{aligned}
J_{x z}^{n+1 / 2} & =\frac{2+\Delta t \sigma_{z_{N}}}{2 \kappa_{z_{N}}+\Delta t\left(\alpha_{z_{N}} \kappa_{z_{N}}+d_{z_{N}}\right)} \Psi_{x z_{N-1}}^{n+1 / 2} \\
& +\frac{2 \kappa_{z_{N}}}{2 \kappa_{z_{N}}+\Delta t\left(\alpha_{z_{N}} \kappa_{z_{N}}+d_{z_{N}}\right)} \Phi_{x z_{N}}^{n-1 / 2}-\frac{\partial \sigma_{x z}^{n+1 / 2}}{\partial Z} .
\end{aligned}
$$

From a computational point of view, it should be noted that $\Psi_{x z_{i}}$ and $J_{x z}$ could be updated within the PML correction loop. This means that they do not have to be stored in the computer memory, thus minimizing the memory requirements. 


\section{Implementation of $\mathbf{N t h}$-order stretching}

Although a formulation for $J_{x z}^{n+1 / 2}$ has now been calculated, it is not yet in an efficient form to facilitate computation. Further analysis reveals that $\Psi_{x z_{i}}$ is merely a function of $\partial \sigma_{x z_{1}} / \partial z, \Phi_{x z_{1}}$, and $\Psi_{x z_{i}}$. This means that all $\Psi_{x z_{i}}$ could be eliminated from equation 42 . The result is written using a single formula describing the correction term $J_{x z}$ at the current time step. This formulation is finally capable of calculating the stretching functions for a PML of arbitrary order:

$$
\begin{aligned}
J_{x z}^{n+1 / 2} & =\left\{\left(\prod_{q=1}^{N} R A_{z_{q}}\right)-1\right\} \frac{\partial \sigma_{x z}^{n+1 / 2}}{\partial z} \\
& +\sum_{i=1}^{n-1}\left\{\left(\prod_{q=i+1}^{N} R A_{z_{q}}\right) R B_{z i} \Phi_{x z}^{n-1 / 2}\right\} \\
& +R B_{z_{N}} \Phi_{x z_{N}}^{n-1 / 2}
\end{aligned}
$$

where $i \in[2, N-1]$.

Similarly, due to the same relationship between $\Psi_{x z_{i}}, \partial \sigma_{x z_{1}} / \partial z$, and $\Phi_{x z_{1}}, \Psi_{x z_{i}}$ is eliminated from the summation memory variable. This yields

$$
\begin{aligned}
\Phi_{x z_{i}}^{n+1 / 2} & =R E_{z_{i}} \Phi_{x z_{i}}^{n-1 / 2}-R F_{z_{i}}\left\{\left(\prod_{q=1}^{i-1} R A_{z_{q}}\right) \frac{\partial \sigma_{x z}^{n+1 / 2}}{\partial Z}\right. \\
& \left.+\sum_{m=1}^{i-1}\left(\prod_{q=m+1}^{i-1} R A_{z_{q}}\right) R B_{z_{m}} \Phi_{x z_{m}}^{n-1 / 2}\right\}
\end{aligned}
$$

where $i \in[2, N-1]$. The $R A_{\mathrm{zi}}, R B_{\mathrm{zi}}, R E_{\mathrm{zi}}, R F_{\mathrm{zi}}$ are defined by

$$
\begin{aligned}
R A_{z_{i}} & =\frac{2+\Delta t \alpha_{z_{i}}}{2 \kappa_{z_{i}}+\Delta t\left(\alpha_{z_{i}} \kappa_{z_{i}}+d_{z_{i}}\right)}, \\
R B_{z_{i}} & =\frac{2 \kappa_{z_{i}}}{2 \kappa_{z_{i}}+\Delta t\left(\alpha_{z_{i}} \kappa_{z_{i}}+d_{z_{i}}\right)}, \\
R E_{z_{i}} & =\frac{2 \kappa_{z_{i}}-\Delta t\left(\alpha_{z_{i}} \kappa_{z_{i}}+d_{z_{i}}\right)}{2 \kappa_{z_{i}}+\Delta t\left(\alpha_{z_{i}} \kappa_{z_{i}}+d_{z_{i}}\right)}, \\
R F_{z_{i}} & =\frac{2 \sigma_{z_{i}} \Delta t}{\left(2 \kappa_{z_{i}}+\Delta t\left(\alpha_{z_{i}} \kappa_{z_{i}}+d_{z_{i}}\right)\right) \kappa_{z_{i}}} .
\end{aligned}
$$

Note that only $J_{x z}^{n+1 / 2}$ and $\Phi_{x z_{i}}^{n+1 / 2}$ requires updating within the computational time loop. To implement them with the PML scheme, they are calculated and updated at each time step and then added to the original FDTD field variables (i.e., equations 14-18). The $R A_{\mathrm{zi}}, R B_{\mathrm{zi}}, R E_{\mathrm{zi}}$, and $R F_{\mathrm{zi}}$ are not time dependent and therefore could be calculated before the commencement of the integration scheme. Note that due to the nature of the PML, the material properties within the PML regions do not require modification and remain identical to those within the non-PML domain. Additionally, the PML corner regions do not require any additional equations or consideration, thus significantly increasing the ease of programming.

\section{First-order implementation}

Equation 43 describes the calculation of the correction term to implement a PML of arbitrary order. If $N=1$, this formulation reduced to a first-order (O1) PML. Note that the first-order PML uses only a single stretching function and mathematically was similar to the one presented by Drossaert and Giannopoulos (2007), albeit with the aforementioned advantages. The formula results in

$$
J_{x z}^{n+1 / 2}=\left\{R A_{\mathrm{z}_{1}}-1\right\} \frac{\partial \sigma_{x z}^{n+1 / 2}}{\partial z}+R B_{\mathrm{z}_{1}} \Phi_{x z_{1}}^{n-1 / 2},
$$

followed by the update of $\Phi_{x z_{1}}^{n+1 / 2}$ as

$$
\Phi_{x z_{1}}^{n+1 / 2}=R E_{\mathrm{z}_{1}} \Phi_{x z_{1}}^{n-1 / 2}-R F_{\mathrm{z}_{1}} \frac{\partial \sigma_{x z}^{n+1 / 2}}{\partial z} .
$$

\section{Second-order implementation}

Second-order (O2) PMLs have been shown to offer increased absorption performance in the field of electromagnetics. Notice that two stretching functions are now required to describe the overall PML attenuation. Inserting $N=2$ into the correction equation 43 results in

$$
\begin{aligned}
J_{x z}^{n+1 / 2} & =\left\{R A_{\mathrm{z}_{1}} R A_{\mathrm{z}_{2}}-1\right\} \frac{\partial \sigma_{x z}^{n+1 / 2}}{\partial z} \\
& +R A_{\mathrm{z}_{2}} R B_{\mathrm{z}_{1}} \Phi_{x z_{1}}^{n-1 / 2}+R B_{\mathrm{z}_{2}} \Phi_{\mathrm{x} z_{2}}^{n-1 / 2},
\end{aligned}
$$

which now depends on two updates, for $\Phi_{x z_{2}}$ and $\Phi_{x z_{1}}$ as

$$
\begin{aligned}
\Phi_{x z_{2}}^{n+1 / 2} & =R E_{\mathrm{z}_{2}} \Phi_{x z_{2}}^{n-1 / 2} \\
& -R F_{\mathrm{z} 2}\left\{R A_{\mathrm{z}_{1}} \frac{\partial \sigma_{x z}^{n+1 / 2}}{\partial z}+R B_{\mathrm{z}_{1}} \Phi_{x z_{1}}^{n-1 / 2}\right\}
\end{aligned}
$$

and

$$
\Phi_{x z_{1}}^{n+1 / 2}=R E_{\mathrm{z}_{1}} \Phi_{x z_{1}}^{n-1 / 2}-R F_{\mathrm{z}_{1}} \frac{\partial \sigma_{x z}^{n+1 / 2}}{\partial z}
$$

Note that although equations 46 and 48 take different forms depending on the order $N$ of the PML (i.e., first, second, .., N), $R A_{z i}$, $R B_{\mathrm{zi}}, R E_{\mathrm{zi}}$, and $R F_{\mathrm{zi}}$ remains unchanged.

\section{PML stability}

Although it is shown by Giannopoulos (2012) that second-order PMLs can provide enhanced performance over first-order PMLs for electromagnetic wave absorption, it is still unclear as to whether PML orders greater than second provided any significant benefit. Therefore, this work focuses primarily upon second-order implementation and testing.

The first-order stretching functions are written as

$$
s_{\text {classical }}=\kappa+\frac{d}{i \omega}
$$


and

$$
s_{\mathrm{CFS}}=\kappa+\frac{d}{\alpha+i \omega} .
$$

The stretching function of a second-order PML depends on the multiplication of two first-order stretching functions. Therefore, considering equations 48 and 6 , there are three possible permutations of the second-order stretching function:

$$
\begin{gathered}
s_{\text {classical-classical }}=\left(\kappa+\frac{d}{i \omega}\right)\left(\kappa+\frac{d}{i \omega}\right), \\
s_{\text {classical-CFS }}=\left(\kappa+\frac{d}{i \omega}\right)\left(\kappa+\frac{d}{\alpha+i \omega}\right), \text { and } \\
s_{\text {CFS-CFS }}=\left(\kappa+\frac{d}{\alpha+i \omega}\right)\left(\kappa+\frac{d}{\alpha+i \omega}\right) .
\end{gathered}
$$

The $s_{\text {classical-classical }}$ stretching function is found to offer poor performance due its limited ability to absorb low-frequency waves and possibly due to the large similarity between first- and second-order stretching components. Therefore, it is discounted from the analysis at an early stage.

The selection of stretching function parameters can have a significant effect on a PML's ability to attenuate wave energy. Careless parameter selection can also lead to instability, especially as the PML order increases. To meet the stability criterion, the real part of the stretching function has to be greater or equal to one or the PML caused a physical contraction of space rather than a stretching of space. Similarly, the imaginary part has to be less than or equal to zero or the PML region experiences a magnification of amplitude rather than an attenuation. These relationships are expressed as

$$
r_{O 2} \geq 1
$$

and

$$
I_{O 2} \leq 0
$$

Different second-order (and $N$ th-order) stretching functions yield different real and imaginary components. Therefore, equations 54 and 55 are analyzed to determine appropriate parameter selections to meet the conditions outlined in equations 56 and 57. Despite this, it should be noted that in cases in which the stability criteria are not met, it does not necessarily guarantee that errors will be introduced into the solution.

\section{Classical-CFS stability criterion}

Giannopoulos (2012) finds that for the absorption of electromagnetic waves, optimum performance is achieved by combining classical and CFS stretching functions (equation 54). It is postulated that for some domains, by combining the classical PMLs ability to absorb frequency-independent waves with the CFS-PMLs ability to absorb low-frequency evanescent waves, greater performance was achievable. For the elastodynamics case, the real and imaginary components of the "classical-CFS" case are found to be

$$
r_{O 2}=\kappa_{2}+\frac{d_{2} \alpha_{2}}{\alpha_{2}^{2}+\omega^{2}}-\frac{d_{1} d_{2}}{\alpha_{2}^{2}+\omega^{2}}
$$

and

$$
I_{O 2}=\frac{d_{1} \kappa_{2}}{\omega}+\frac{d_{2} \omega}{\alpha_{2}^{2}+\omega^{2}}+\frac{d_{1} d_{2} \alpha_{2}}{\omega\left(\alpha_{2}^{2}+\omega^{2}\right)} .
$$

\section{CFS-CFS stability criterion}

The CFS-CFS PML approach had a greater number of degrees of freedom in comparison with the RI-CFS-PML because additional $\alpha$ and $\kappa$ coefficients were used. In fact, the classical-CFS formulation can be considered as a subset of the CFS-CFS formulation. Therefore, the CFS-CFS PML is likely to provide enhanced absorption.

The real and imaginary parts of the CFS-CFS stretching function are found to be

$$
\begin{aligned}
r_{O 2}= & \kappa_{1} \kappa_{2}+\frac{\alpha_{2} \kappa_{1} d_{2}}{\alpha_{2}^{2}+\omega^{2}}+\frac{\alpha_{1} \kappa_{2} d_{1}}{\alpha_{1}^{2}+\omega^{2}} \\
& +\left(\frac{\alpha_{1} \alpha_{2}}{\left(\alpha_{1}^{2}+j \omega^{2}\right)\left(\alpha_{2}^{2}+j \omega^{2}\right)}-\frac{\omega^{2}}{\left(\alpha_{1}^{2}+j \omega^{2}\right)\left(\alpha_{2}^{2}+j \omega^{2}\right)}\right)
\end{aligned}
$$

and

$$
\begin{aligned}
I_{O 2}= & \frac{\kappa_{1} \omega d_{2}}{\left(\alpha_{2}^{2}+j \omega^{2}\right)}+\frac{d_{1} \omega \kappa_{2}}{\left(\alpha_{1}^{2}+j \omega^{2}\right)} \\
& -\left(\frac{\alpha_{2} \omega}{\left(\alpha_{1}^{2}+\omega^{2}\right)\left(\alpha_{2}^{2}+\omega^{2}\right)}+\frac{\alpha_{1} \omega}{\left(\alpha_{1}^{2}+\omega^{2}\right)\left(\alpha_{2}^{2}+\omega^{2}\right)}\right) d_{1} d_{2} .
\end{aligned}
$$

Assuming that all PML coefficient values are chosen to be positive, the imaginary part of the stretching function would always be fulfilled. Despite this, unlike the classical-CFS case, the stability of the real part of the CFS-CFS stretching function is frequency dependent. Therefore, to maintain stability, $\alpha_{1} \alpha_{2}>\omega^{2}$ and $\kappa_{2} \geq 1$.

Unlike the classical-CFS stretching function, $\alpha_{2}$ scaling has to be considered relative to $\alpha_{1}$ scaling to ensure that the stability criteria are met at every grid point within the PML region. If $\alpha_{1}$ and $\alpha_{2}$ are scaled from a minimum at the threshold between PML and modeling space, to a maximum at the PML extremity, then it is more straightforward to ensure that the stability condition is met.

Although the CFS-CFS stability criterion is slightly more challenging to meet, in comparison with the classical-CFS case, it offers five more degrees of freedom to aid absorption.

\section{NUMERICAL RESULTS}

\section{Example 1: Square 2D homogeneous full-space}

\section{Problem formulation}

The performance of the new correction PML formulation in comparison with a traditional noncorrection approach is compared using a square 2D homogeneous full space, as also used by Drossaert and 
Giannopoulos (2007). The domain is $77.5 \mathrm{~m}$ in length in the $x$ - and $z$-directions with a cell size of $0.25 \mathrm{~m}$ (Figure 1). A rotated staggered grid (RSG) stencil is used to describe the staggered nature of the velocity and stress components. The central differencing time integration scheme is second-order accurate in space and time. The material properties are $\rho=2000 \mathrm{~kg} / \mathrm{m}^{3}, \lambda=500 \mathrm{MPa}$, and $\mu=300 \mathrm{MPa}$. The central grid point $(155,155)$ is excited using a pulse in the shape of the second derivative of a Gaussian, with frequency of $20 \mathrm{~Hz}$. The receiver is offset from the upper left corner by $5 \mathrm{~m}$ in both directions.

\section{Model results - Correction RI-PML versus noncorrection RI-PML}

To compare the performance of the new correction PML, its performance was tested against an alternative PML implementation, using example 1. To create the alternative, first-order PML scheme, we follow Drossaert and Giannopoulos (2007). To create the correction PML, equations 46 and 47 are used. A key difference in the approaches is that the noncorrection formulation required direct manipulation of the underlying velocity/stress update equations. The correction PML equations does not require such manipulation and takes the time-domain form:

$$
\begin{gathered}
v_{x}=b\left(\frac{\partial \sigma_{x x}}{\partial x}+\frac{\partial \sigma_{x z}}{\partial z}\right)+\mathrm{PML}_{v_{\mathrm{x}}}, \\
v_{z}=b\left(\frac{\partial \tilde{\sigma}_{x z}}{\partial x}+\frac{\partial \sigma_{z z}}{\partial z}\right)+\mathrm{PML}_{v_{z}}, \\
\sigma_{x x}=(\lambda+2 \mu) \frac{\partial v_{x}}{\partial x}+\lambda \frac{\partial v_{z}}{\partial z}+\mathrm{PML}_{\sigma_{x x}}, \\
\sigma_{z z}=(\lambda+2 \mu) \frac{\partial v_{z}}{\partial z}+\lambda \frac{\partial v_{x}}{\partial x}+\mathrm{PML}_{\sigma_{z z}},
\end{gathered}
$$

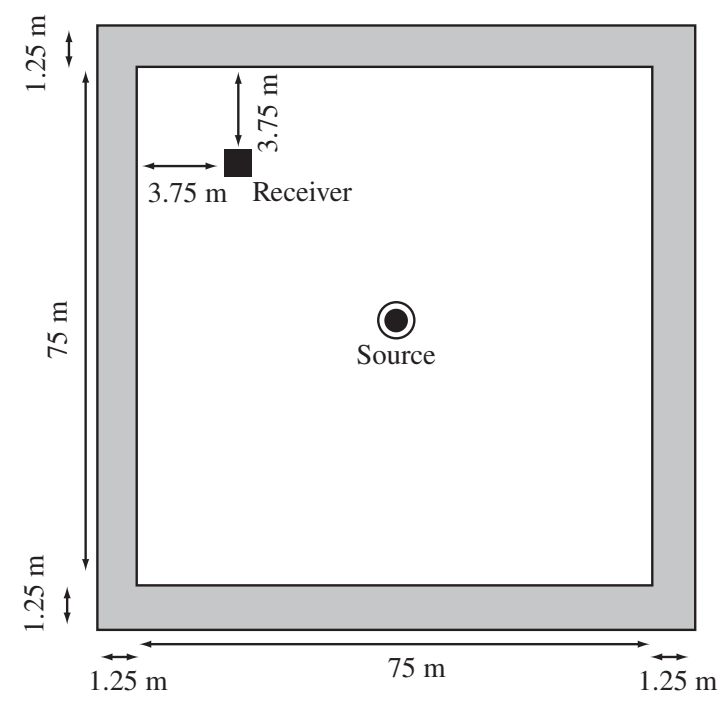

Figure 1. Example 1 schematic.

$$
\sigma_{x z}=\mu\left(\frac{\partial v_{z}}{\partial x}+\frac{\partial v_{x}}{\partial z}\right)+\mathrm{PML}_{\sigma_{x z}}
$$

with the correction coefficients calculated in the PML zones as

$$
\begin{gathered}
\mathrm{PML}_{v_{x}}=b\left(J_{x x}+J_{x z}\right), \\
\mathrm{PML}_{v_{z}}=b\left(J_{x z}+J_{z z}\right), \\
\mathrm{PML}_{\sigma_{x x}}=(\lambda+2 \mu) M_{x x}+\lambda M_{x z}, \\
\mathrm{PML}_{\sigma_{z z}}=(\lambda+2 \mu) M_{z z}+\lambda M_{z x}, \\
\mathrm{PML}_{\sigma_{x z}}=\mu\left(M_{z x}+M_{x z}\right) .
\end{gathered}
$$

The $J_{x u}$ and $M_{x u}$ are calculated as described by equations 19 and 20. The regions for the calculation of the PML correction terms could also be written as

$$
\begin{gathered}
\mathrm{PML}_{u_{i}} \neq 0 \quad \text { for } x \in[0-1.25,76.25-77.5], \\
z \in[0-1.25,76.25-77.5], \\
\mathrm{PML}_{u_{i}}=0 \quad \text { for } x \in[1.25-76.25], \text { and } \\
z \in[1.25-76.25] .
\end{gathered}
$$

For both tests, the PML is five cells thick, and for simplicity, firstorder classical stretching functions (equation 51) are compared (i.e., $\kappa_{\max }=1$ and $\left.\alpha_{\max }=0\right)$. The $d_{\max }$ is calculated in accordance with Collino and Tsogka (2001), and $d$ is scaled quadratically with $d_{\max }$ located at the extremity of the computational grid. The calculation of $d_{\max }$ is shown in equation 76 , where $V_{\mathrm{P}}$ is the compressional wave velocity, $L$ is the PML depth, and $R$ is the reflection coefficient $\left(1 \times 10^{-5}\right)($ Collino and Tsogka, 2001). It should also be noted that the RSG was found to make the implementation of both the PMLs more straightforward than other grid types due to the colocation of velocity components and the colocation of stress components as

$$
d_{\max }=\frac{3 v_{p}}{2 L} \log \frac{1}{R}
$$

Figure 2 shows the resulting traces for both schemes. It was found that the performance of both PMLs was so similar that the trace time histories were not useful for enabling comparison. Therefore, the difference between each trace at each time step was also plotted and is shown in Figure 3. It can be seen that the error was on the order of $1 \times 10^{-15}$, with a maximum of $2 \times 10^{-15}$. Last, a commonly used error metric (equation 77) was used to calculate the difference between traces. Figure 4 shows that the resulting error is in the region of $-300 \mathrm{~dB}$ as 


$$
\text { Error }\left._{\mathrm{db}}\right|_{i, j} ^{n}=20 \log _{10} \frac{\left\|\left.E_{\text {correction }}\right|_{i, j} ^{n}-\left.E_{\text {noncorrection }}\right|_{i, j} ^{n}\right\|}{\left\|E_{\text {noncorrection }_{\max }} \mid{ }_{i, j}\right\|} .
$$

Note that $\left.E_{\text {correction }}\right|_{i, j} ^{n}$ represents the correction PML trace at a point in time $n$ and at spatial location $i, j$. The $\left.E_{\text {noncorrection }}\right|_{i, j} ^{n}$ represents the noncorrection PML solution, and $E_{\text {noncorrection }_{\max }}$ is the maximum amplitude of the noncorrection PML trace. When plotted, this allows for a better visual interpretation of the errors at each point in time.

Therefore, it is concluded that the new correction implementation performed nearly identically to the original noncorrection PML. The discrepancies are in the range of $1 \times 10^{-15}$ or $-300 \mathrm{~dB}$, which, although theoretically they should have been zero, were insignificant and most likely generated by numerical precision errors (e.g., rounding errors associated with the computer, etc.).

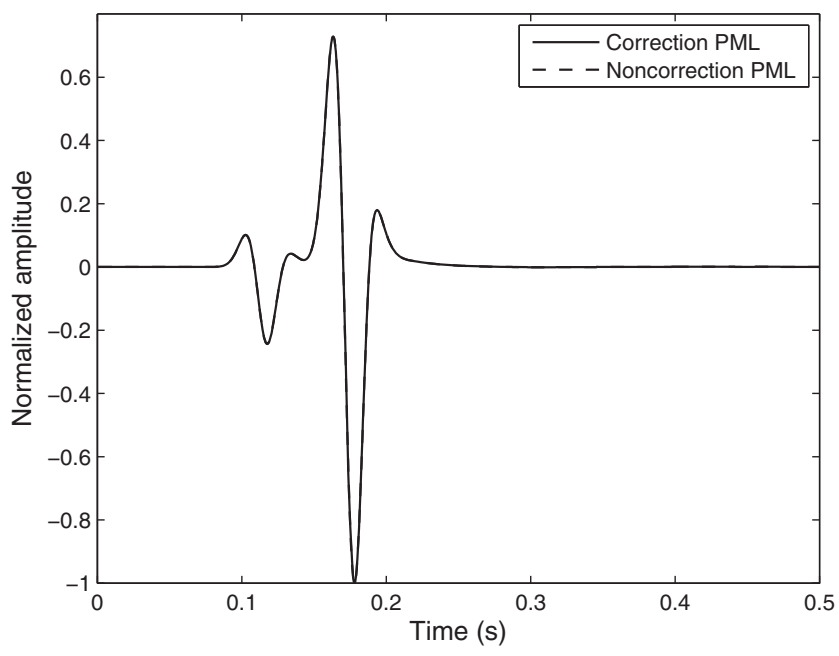

Figure 2. Example 1: Trace history.

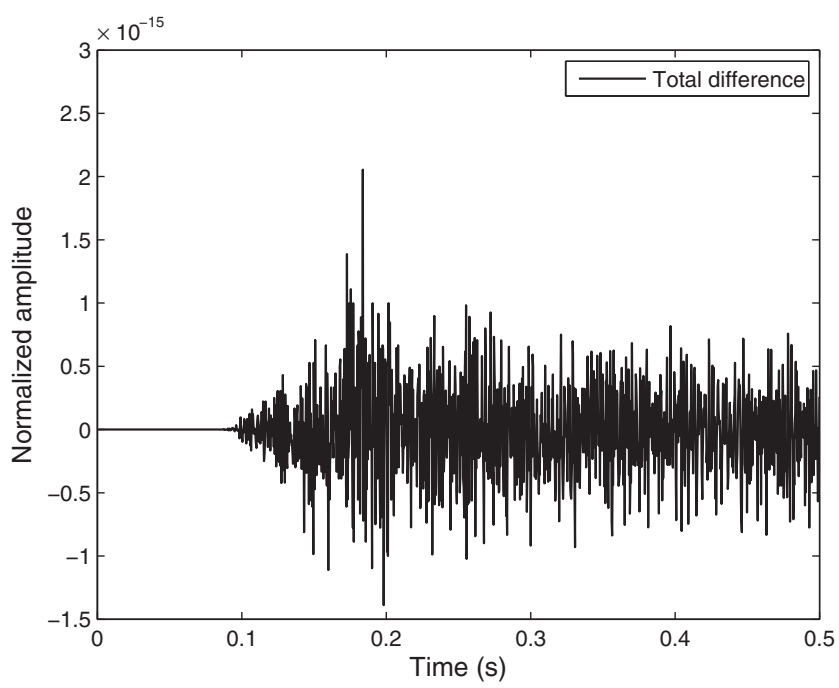

Figure 3. Example 1: Trace error time history.

\section{Example 2: Rectangular 2D homogeneous full-space}

\section{Problem formulation}

Elongated rectangular domains can reduce the absorption performance of PMLs. Therefore, two PML comparisons are undertaken. First, the new first-order recursive integration correction PML is compared with an alternative PML implementation based on a convolution approach. Second, the new higher order correction PML is compared with a first-order correction PML approach.

The grid used for comparison is identical to that outlined in Komatitsch and Martin (2007) and Martin et al. (2010) with $101 \times 641$ square cells and $10-\mathrm{m}$ spacing between grid points in both directions (Figure 5). The homogeneous material is characterized by pressure wave velocity $c_{p}=3300 \mathrm{~ms}^{-1}$, S-wave velocity $c_{s}=1905 \mathrm{~ms}^{-1}$, and density $\rho=2800 \mathrm{kgm}^{-3}$. The computational scheme is second-order accurate in space and time with a constant time step of $\delta t=0.001 \mathrm{~s}$. The staggered grid stencil follows that outlined by Virieux (1986) and is bounded on all sides by a PML region 10 cells thick.

An 8-Hz excitation with the form of a first derivative of a Gaussian was used to excite the velocity components in the horizontal and vertical directions at coordinate $(79,427)$. Receivers one, two, and three were placed at $(20,413),(70,227)$, and $(81,27)$, respectively. Physically, receiver 1 was located closest to the source and receiver 3 located furthest away. At each receiver, horizontal and vertical velocity time histories were recorded.

\section{Example 2 (test 2.1): Correction RI-PML versus C-PML}

For the domain shown in Figure 5, Martin et al. (2010) find a set of high-performance absorption coefficients. Values for $\kappa_{\max }$ and $\alpha_{\max }$ are shown in equations 78 and $79(\omega=$ excitation frequency), and $\alpha$ is scaled linearly with a maximum at the edge of the computational grid (Festa and Vilotte, 2005). The $d_{\max }$ and the profile for $d$ are calculated using the recommendations outlined in Collino and Monk (1998). Therefore, the same coefficients are for the first-order correction PML, based on a recursive integration approach and for the C-PML:

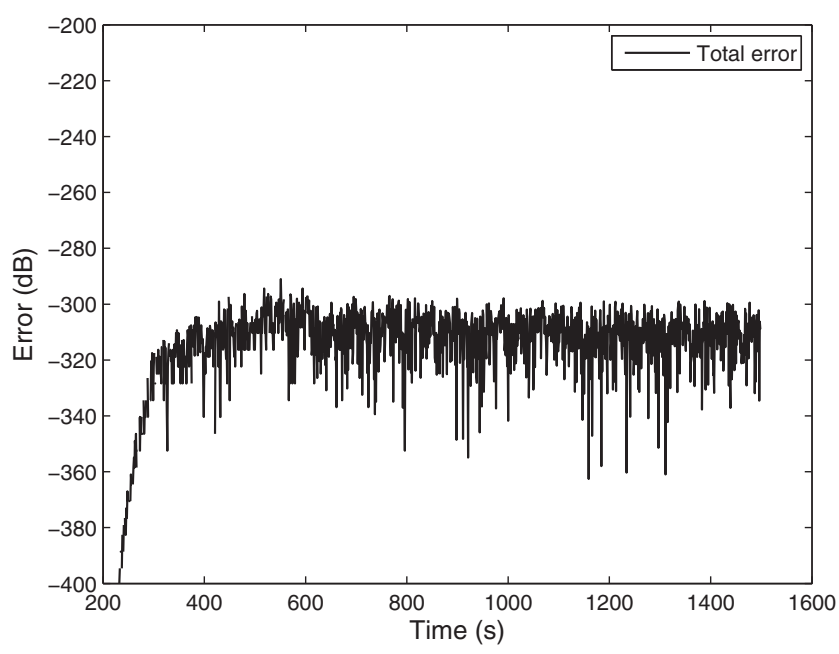

Figure 4. Example 1: Error time history. 


$$
\kappa_{\max }=7 \quad(\alpha \max =\omega \pi) .
$$

Figure 6 shows that the resulting traces for both PML conditions were similar. Therefore, once again, to facilitate a more detailed comparison of performance, a log-based metric is introduced. Note that this time, the error is calculated for the performance of each PML compared with a reference solution, rather than directly between the PML formulations:

$$
\text { Error }\left._{\mathrm{db}}\right|_{i, j} ^{n}=20 \log _{10} \frac{\left\|\left.E\right|_{i, j} ^{n}-\left.E_{\mathrm{ref}}\right|_{i, j} ^{n}\right\|}{\left\|\left.E_{\mathrm{ref}_{\max } \mid}\right|_{i, j}\right\|} .
$$

Although the error plots (Figure 7) allow for easier comparison between traces, there are still large similarities between results. At some points, the CPML is found to perform marginally better, but at other points, the correction PML exhibits slightly higher accuracy. This is evident at all receivers. Therefore, it is concluded that the correction PML offers very similar performance to the CPML implementation. Despite this, due to the recursive integration scheme used for the RIPML, it offers a solution with reduced memory requirements.

\section{Example 2 (test 2.2): First-order PML versus second-order PML}

\section{A practical approach to optimization}

An advantage of using a higher order PML in comparison with a first-order PML was that there were a greater number of parameters that could be altered to optimize absorption. A challenge associated with these extra degrees of freedom is that each one has to be carefully chosen to maximize absorption and meet stability criteria. This task can be time-consuming.

For example, first considering the stretching function associated with the original first-order classical PML (equation 51), it has only two modifiable degrees of freedom: $d_{\max }$ and the polynomial scaling order for $d$. This makes the optimization process relatively straightforward. In comparison, the alternative CFS-PML stretching function has seven degrees of freedom, including $d_{\max }, \kappa_{\max }, \alpha_{\max }$, and polynomial scaling of $d, \kappa, \alpha$. Additionally, assuming that $d$ and $\kappa$ are always scaled with a maximum at the edge of the computational grid, but $\alpha$ could be scaled in either direction, this creates one extra degree of freedom. This degree of freedom describes the scaling direction.

Then, considering higher order stretching functions, it is clear that the number of degrees of freedom would increase even further. For example, regarding a second-order PML, the classical-CFS PML stretching function (equation 54) has nine degrees of freedom and the CFS-CFS stretching function (equation 55) has 14 degrees

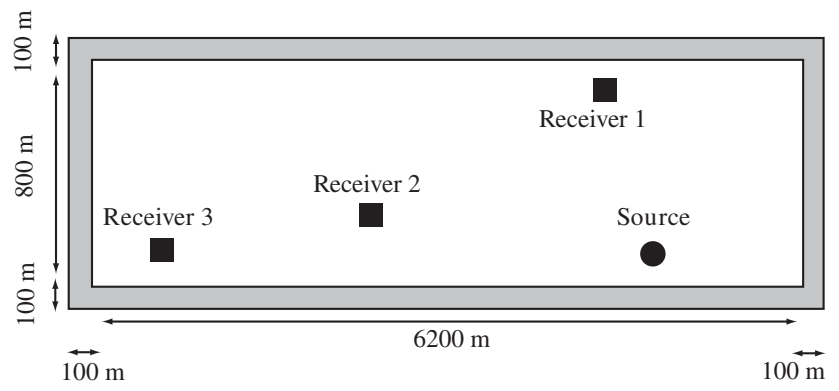

Figure 5. Example 2: Model schematic (rotated $90^{\circ}$ ). of freedom. Therefore, it becomes clear that the optimization task required for the overall stretching function is onerous. If $N_{\text {dof }}=$ number of degrees of freedom within a stretching function, then the total number of dof's needed to describe a PML made up from a number Classical $_{n}$ of classical stretching functions and a number $\mathrm{CFS}_{n}$ of CFS stretching functions is

$$
N_{\text {dof }}=2 \text { Classical }_{n}+7 \mathrm{CFS}_{n} .
$$

The aim of this work is to present the new formulations for the first-order correction PML and the higher order PML. It is also to show that the higher order PML formulation was capable of offering absorption benefits over traditional first-order approaches. Despite this, it is not intended to investigate approaches to the optimization of higher order PML coefficients — instead, as an initial investigation, a trial-and-error approach to optimization is used exclusively (for all examples presented in this work). PML coefficient optimization is an active and ongoing area of research, and as such, it is anticipated that new developments will allow for the efficient optimization of PML coefficients, thus increasing the performance and competitiveness of higher order formulations in comparison with the first-order PMLs.

Therefore, an attempt is made to show that the higher order PML is capable of outperforming first-order schemes when minimizing the number of degrees of freedom required for optimization. This is achieved by using test examples from the literature in which the original authors already determine the optimum first-order parameters. These parameters are then combined with a second set of CFS parameters to create a second-order CFS-CFS stretching function. This means that the higher order PML performance could be investigated when optimizing only a reduced number of coefficients. It also provides a conclusive method to test whether the second-order PML offered enhanced performance over the first-order PML.

\section{Second-order PML versus first-order PML}

To test the ability of a higher order PML scheme to outperform its first-order counterpart, the example outlined by Martin et al. (2010) is also used. The original optimized stretching function coefficients as outlined by Martin et al. (2010) are used to describe the first-order CFS stretching component. This is then combined with another CFS stretching function, as described by equation 55 to create a second-order CFS-CFS stretching function. The time-domain equations are identical to those presented in equations 62-66; however, the correction PML terms (i.e., $J_{x u}$ ) are modified to include the second-order CFS-CFS stretching function (equations 48 and 55).

The second set of CFS parameters are as follows:

$$
\begin{gathered}
d_{\max _{2}}=\frac{d_{\max _{1}}}{30}, \\
\kappa_{\max _{2}}=1.5, \\
\alpha_{\max _{2}}=2 d_{\text {max }_{1}} .
\end{gathered}
$$

It should also be noted that $d_{2}$ and $\kappa_{2}$ are scaled using second-order polynomials and that $\alpha_{2}$ was scaled linearly.

Figure 8 shows the resulting error plots. Both of the first-order CFS formulations produce nearly identical results, and it is found 
a)

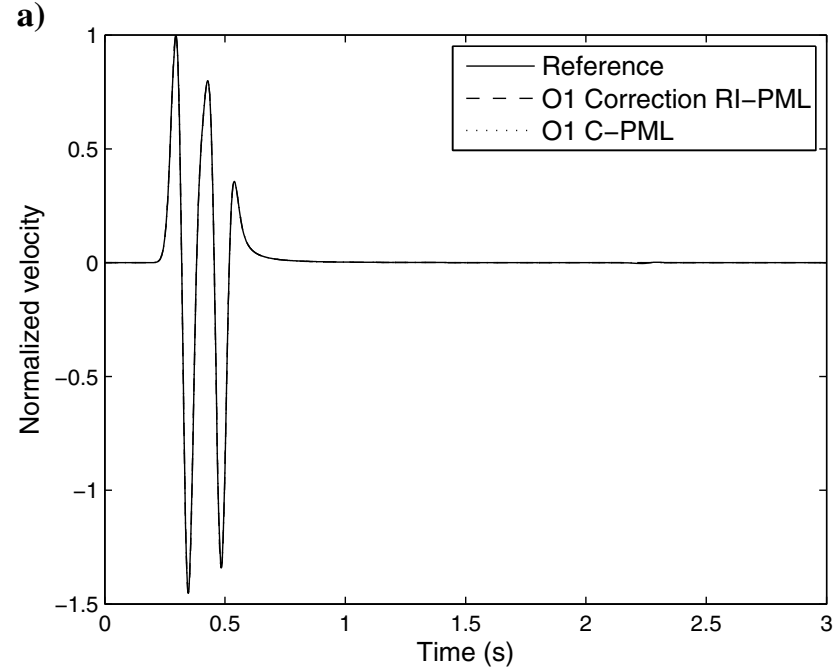

c)

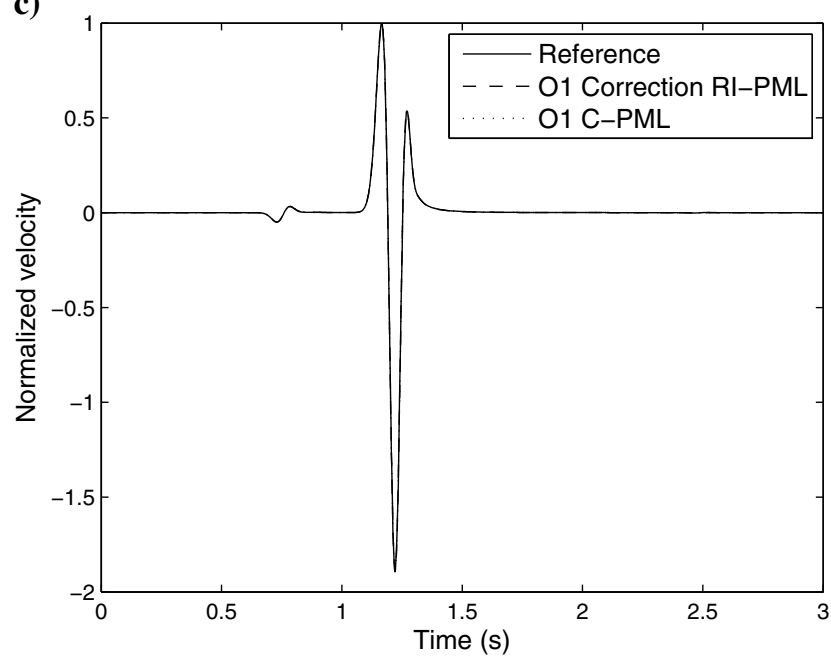

e)

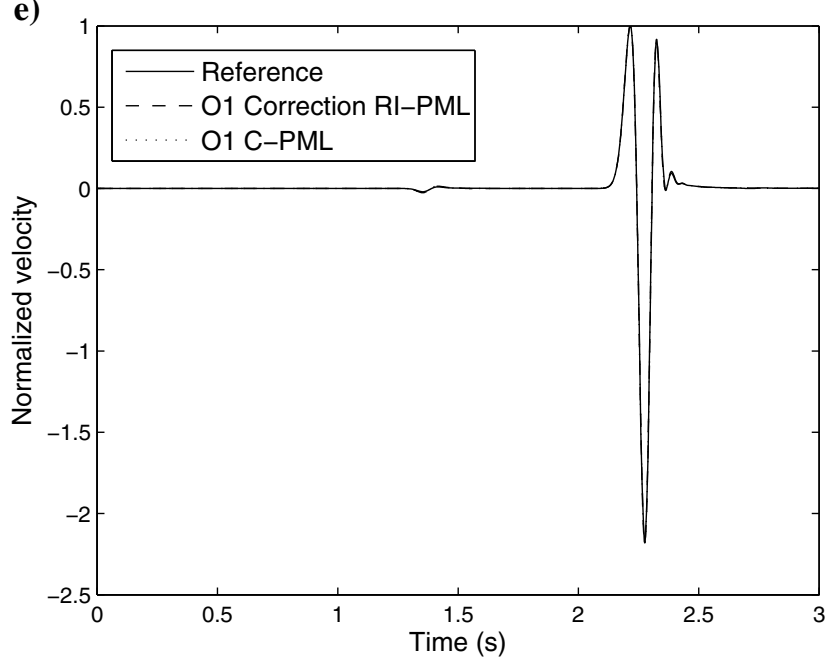

b)

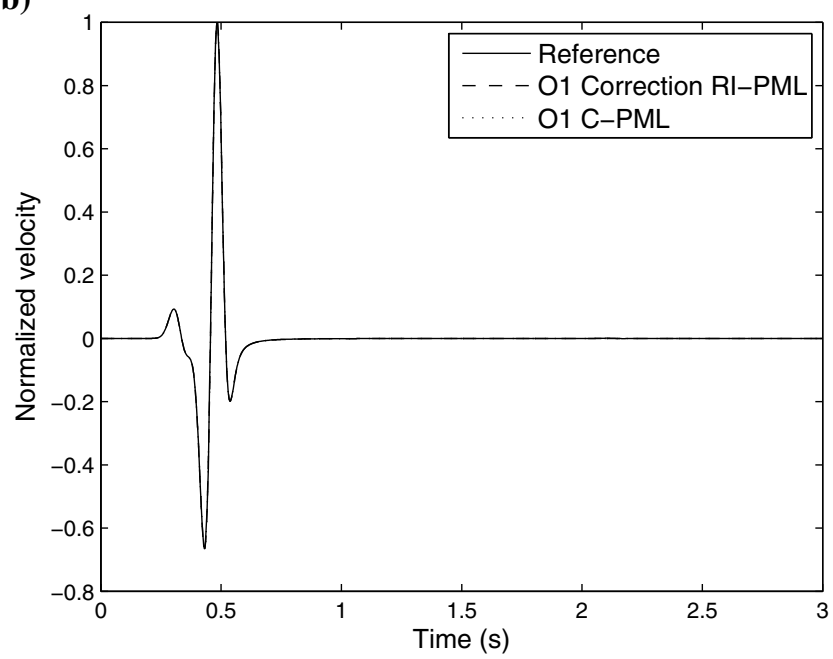

d)

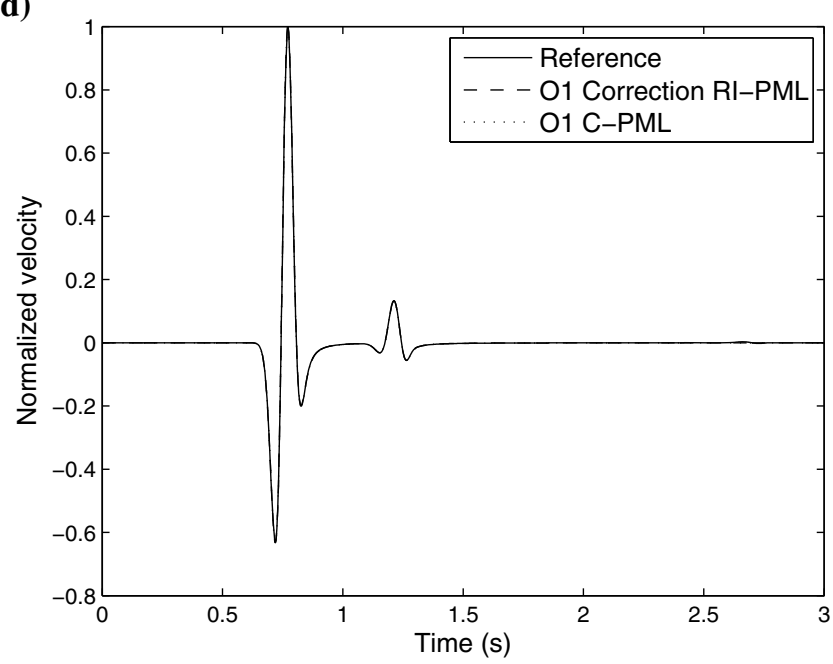

f)

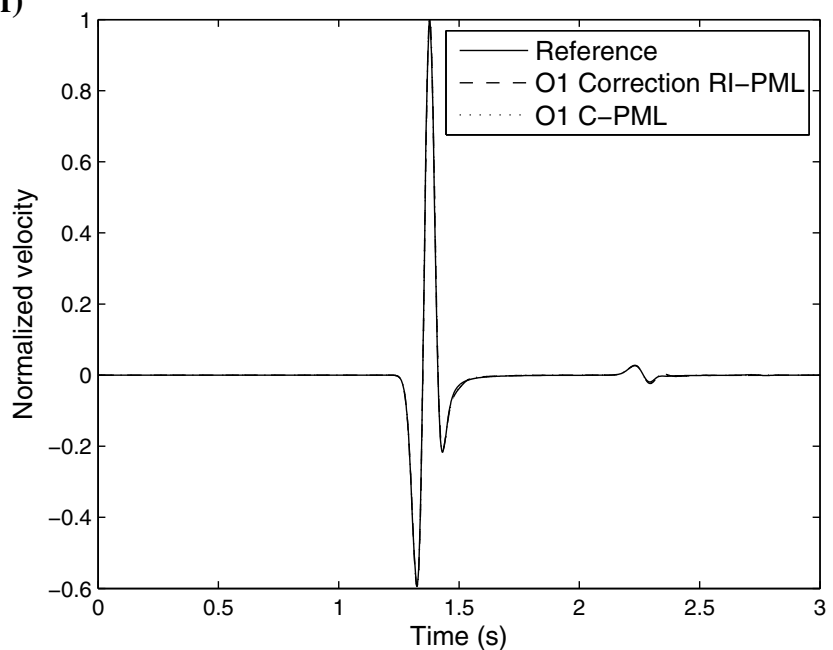

Figure 6. Trace comparisons: RI-PML versus C-PML. (a) $\nu_{x}$ receiver 1 , (b) $\nu_{z}$ receiver 1 , (c) $\nu_{x}$ receiver 2 , (d) $\nu_{z}$ receiver 2 , (e) $\nu_{x}$ receiver 3 , and (f) $\nu_{z}$ receiver 3 . 
that the overall error increases as the receiver distance is increased. Concerning the $\mathrm{O} 2 \mathrm{CFS}-\mathrm{CFS}$ implementation, performance at receivers $v x_{1}$ and $v z_{1}$ is improved slightly but as distance increased,
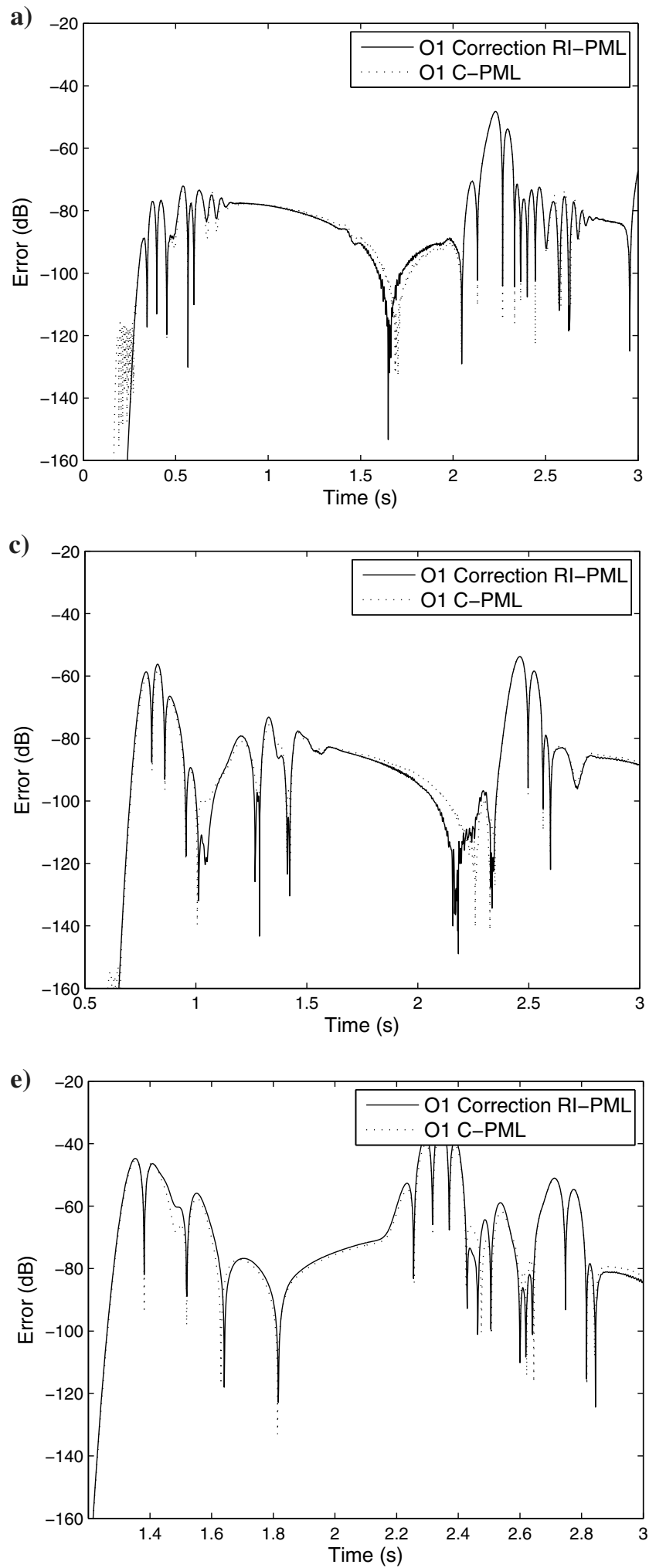

the performance benefit increases rapidly in comparison with the O1 scheme. Receivers $v x_{3}$ and $v z_{3}$ show marked improvement with, on average, between 10 and $20 \mathrm{~dB}$ less error.

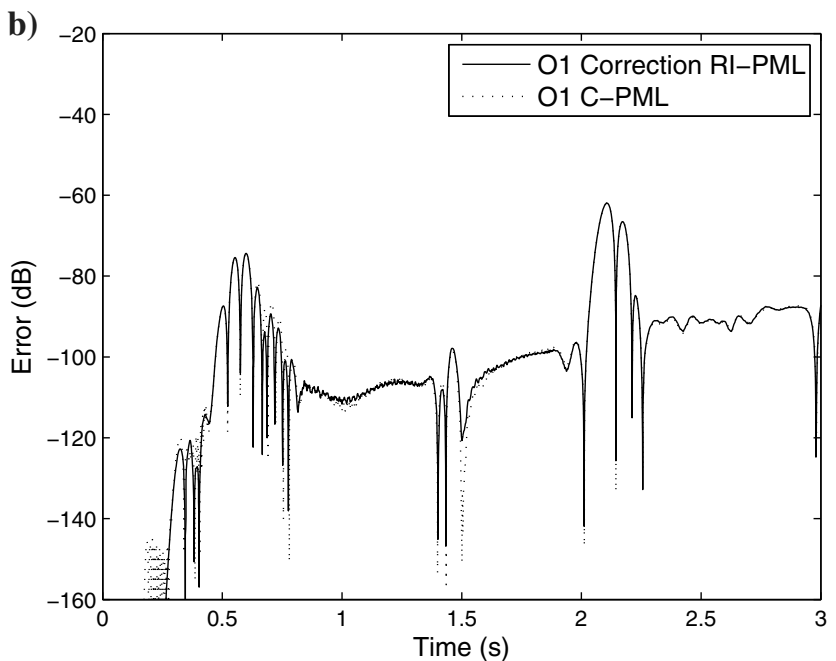

d)
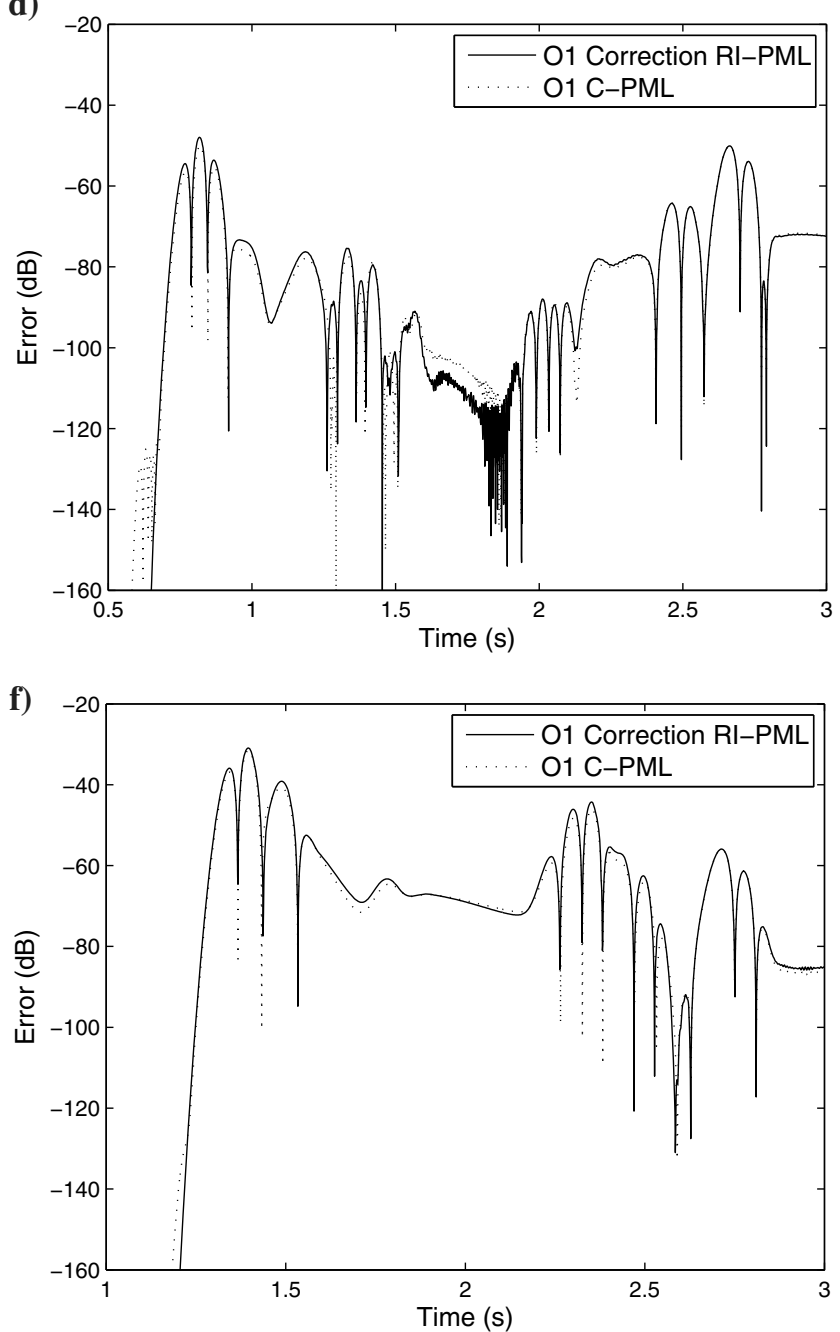

Figure 7. Error comparisons: RI-PML versus C-PML. (a) $\nu_{x}$ receiver 1 , (b) $\nu_{z}$ receiver 1 , (c) $\nu_{x}$ receiver 2 , (d) $\nu_{z}$ receiver 2 , (e) $\nu_{x}$ receiver 3 , and (f) $\nu_{z}$ receiver 3 . 
The furthest-away receivers are subject to a greater number of evanescent waves in comparison with the closest receivers. PML schemes typically have degraded performance under such condi-

a)
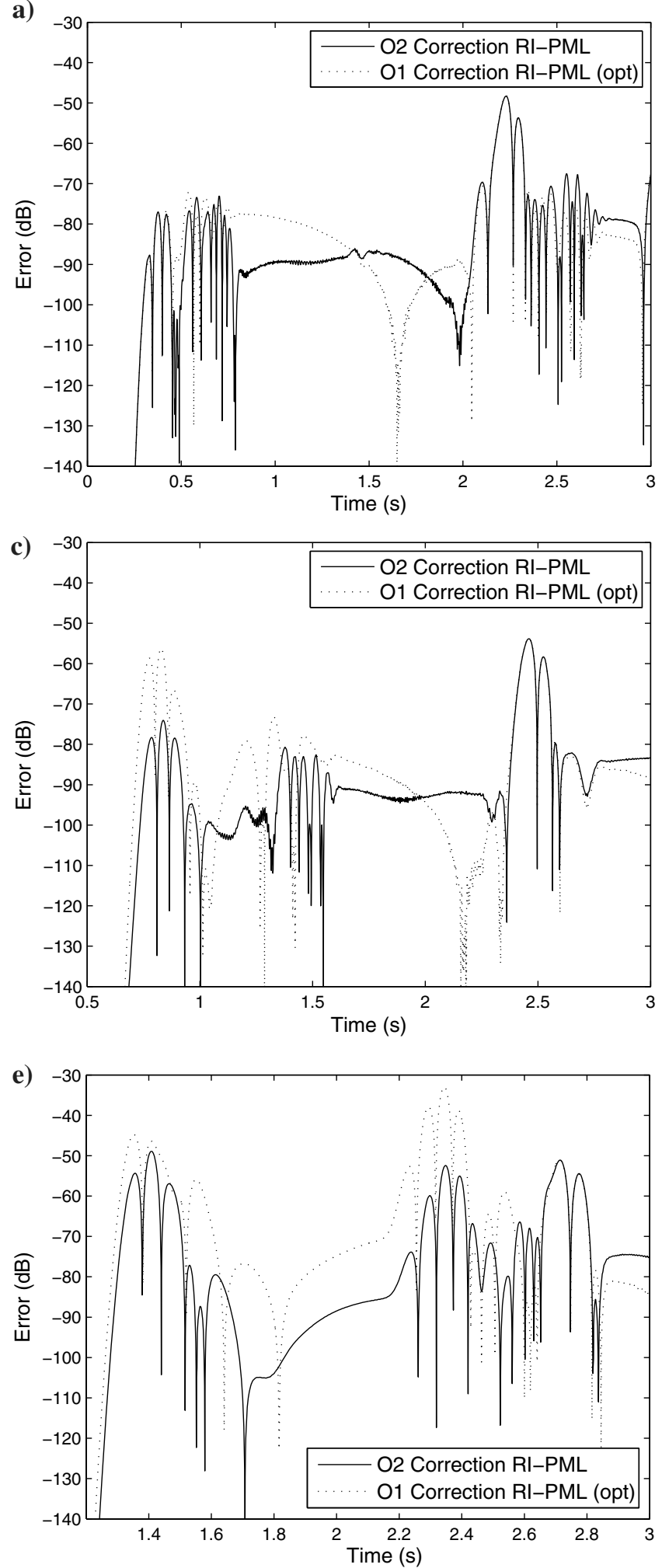

tions, but the additional degrees of freedom associated with the O2 PML allow it to maintain higher levels of performance in comparison with both the CFS stretching functions.

b)
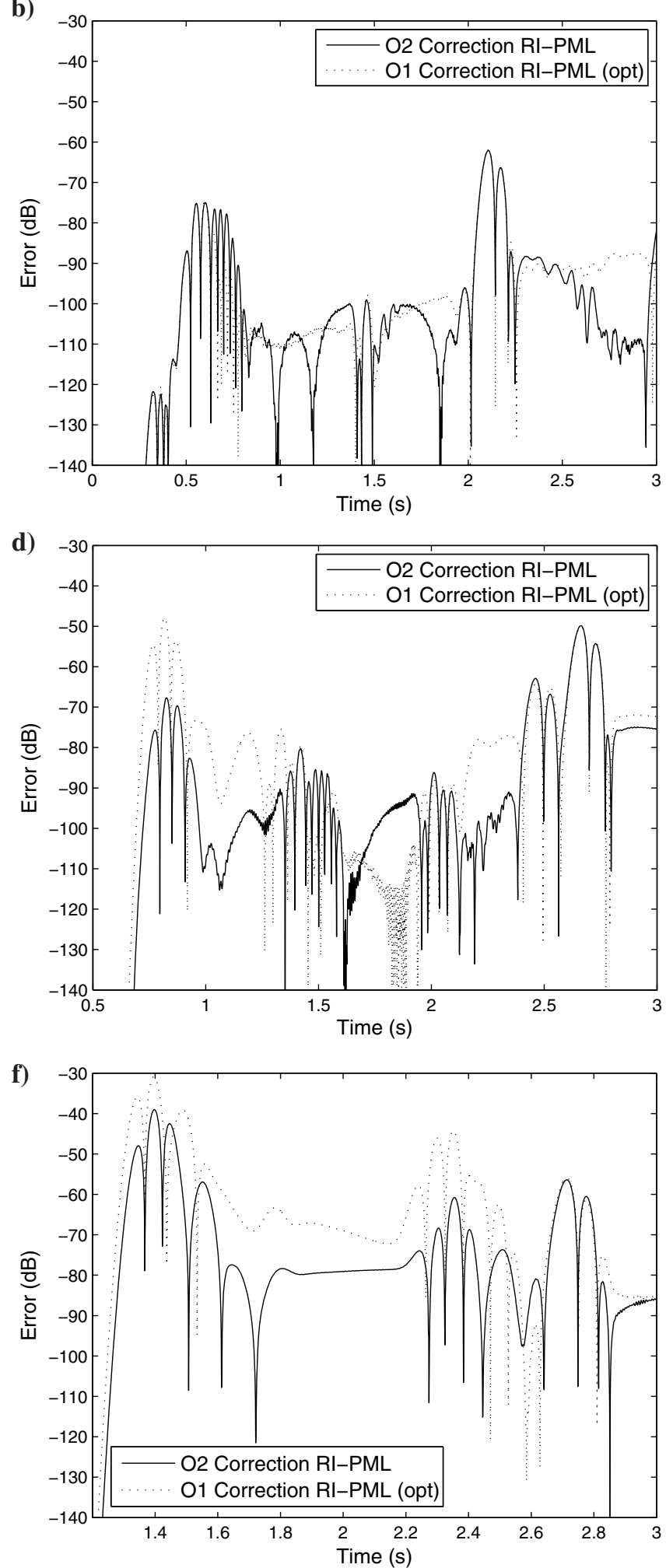

Figure 8. Error comparisons: First-order PML versus second order PML. (a) $\nu_{x}$ receiver 1 , (b) $\nu_{z}$ receiver 1 , (c) $\nu_{x}$ receiver 2, (d) $\nu_{z}$ receiver 2 , (e) $\nu_{x}$ receiver 3 , and (f) $\nu_{z}$ receiver 3 . 
Because the close receivers experienced only a low percentage of evanescent waves, the first-order CFS is also capable of high-performance absorption. Therefore, there is not much scope for improvement by adding an additional stretching function.

It should be noted that attempts were also made to improve absorption performance using the classical-CFS stretching function. Despite this, for this particular full-space model, no significant performance benefits were found.

Investigations were also performed to compare the computational performance between the first- and second-order PMLs. Simulations were performed using MATLAB, running on a laptop $\mathrm{PC}$ with a 2.66-GHz processor and 4 GB of RAM. The computational time taken for the first-order test case to run was $91 \mathrm{~s}$, whereas the second-order test case took $140 \mathrm{~s}$. Therefore, when considering the deployment of a higher order PML, it is important that the potential absorption benefit justifies the increase in computational resource. One possible application is the use of a hybrid (01-02) PML in which all model edges except those subject to a large percentage of evanescent waves are subject to a first-order PML, whereas the remaining sides are terminated using a secondorder PML. Depending on the domain under consideration, this potentially has the ability to offer increased absorption with minimized run times.

Another consideration is that programming the extra equations/ fields associated with the higher order PML requires additional time and effort. Thus, it must also be considered whether this extra effort is better spent modifying an existing first-order implementation (e.g., increasing the physical thickness of the PML), rather than programming a higher order PML.

Therefore, in conclusion, due to the increased performance, particularly at large offsets, the second-order PML offers additional absorption in comparison with its first-order alternative. Despite this, the second-order PML requires additional computational resources and programming effort.

\section{Example 3: A rectangular 2D homogeneous half-space}

\section{Test 3.1: Second-order PML versus first-order PML}

For relatively near-surface seismic applications such as earthquake engineering, it is common to perform simulations in the presence of a free-surface boundary condition. Such models generate wavefields that are more complex than full-space models. These wavefields include surface waves in the form of exponentially decaying Rayleigh waves, which can present challenges for ABCs (Zeng et al., 2011).

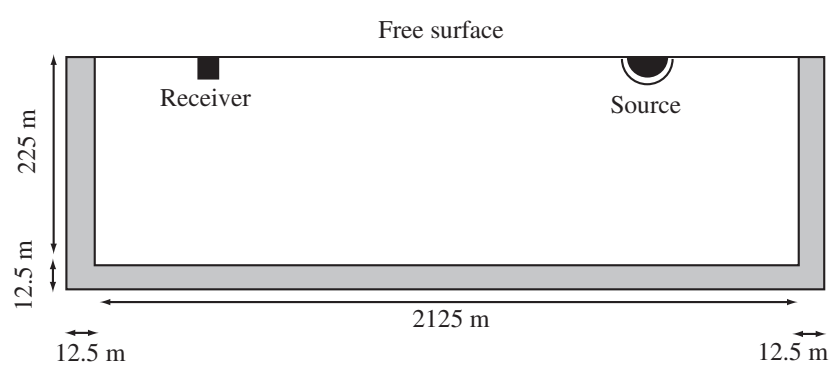

Figure 9. Example 3: Model schematic.
To test the ability of the higher order PML to absorb surface waves, a near-surface model was replicated from Drossaert and Giannopoulos (2007). This elongated domain had dimensions of $3750 \times 250 \mathrm{~m}$ with cell size of $2.5 \mathrm{~m}$ and a time step of $3.2 \mathrm{~ms}$. The source and receiver were placed $2125 \mathrm{~m}$ apart to test the ability of the PML to absorb evanescent waves subject to large grazing angles.

The free surface was achieved by setting the density and S-wave velocity of the five cells above the free surface close to zero. An RSG, with second-order accuracy in space and time, was used to describe the velocity and stress discretization. The use of the RSG facilitated a straightforward implementation of the free surface because only density values had to be averaged across the interface. Excitation was provided by a Gaussian-shaped impulse (center frequency $1.5 \mathrm{~Hz}$ ) in the vertical direction. The material properties of the medium were $\rho=2000 \mathrm{~kg} / \mathrm{m}^{3}, \lambda=$ $600 \mathrm{MPa}$, and $\mu=300 \mathrm{MPa}$.

The PML zones truncated three sides of the grid, with the vertical sides terminating at the free surface as shown in Figure 9. The optimal PML coefficients for the domain, as previously found by Drossaert and Giannopoulos (2007) are $\sigma_{\max }=234.2, \kappa_{\max }=$ 25, and $\alpha_{\max }=10$. All attenuation parameters are scaled using a second-order polynomial function; however, $\alpha_{\max }$ is scaled inversely, meaning it has a value of zero at the extremity of the grid. Once again, a large reference model is used to assess PML performance.

A stretching function with form $s_{\mathrm{CFS}-\mathrm{CFS}}$ is used with parameters

$$
\begin{gathered}
d_{\max _{2}}=50 d_{\max _{1}}, \\
\kappa_{\max _{2}}=1, \\
\alpha_{\max _{2}}=800 .
\end{gathered}
$$

Note that $\alpha_{2}$ is scaled linearly, also with a minimum at the extremity of the grid. The value of $d_{2}$ is scaled in the same manner as $d_{1}$.

Figure 10 shows the resulting vertical velocity error for the surface receiver. It was found that the maximum error occurred

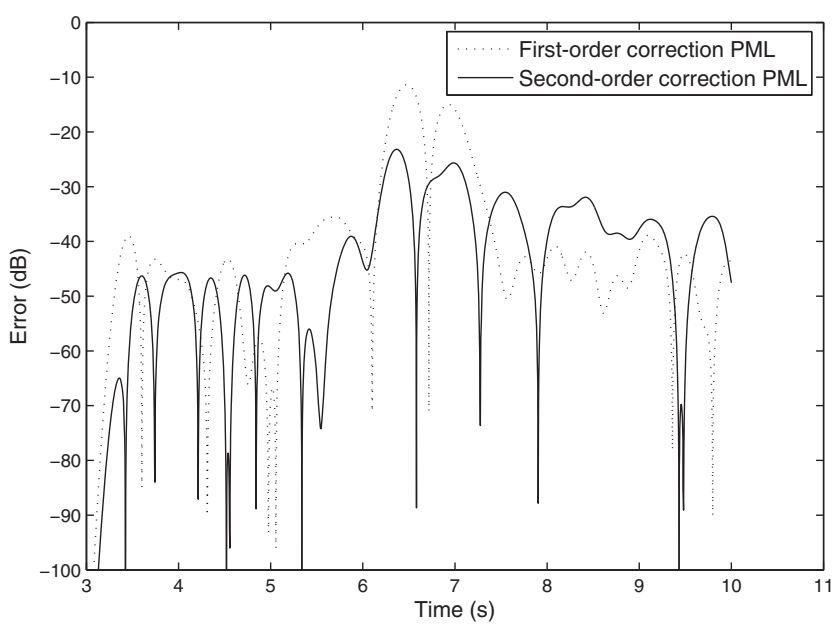

Figure 10. Example 3: Error. 
approximately at $6.5 \mathrm{~s}$ with the first-order PML generating an error of $-11.5 \mathrm{~dB}$. In comparison, the second-order PML reduced this error by $-11.5 \mathrm{~dB}$ to a total of $-23 \mathrm{~dB}$. At the time steps before the maximum error, the second-order PML was found to offer a slight improvement in performance, whereas after the maximum error, it was found to offer a slight decrease in absorption. Despite this, the overall error was lower and the error profile was flatter indicating an overall improvement in performance.

\section{CONCLUSIONS}

Two new unsplit PML formulations were presented for the absorption of seismic wave energy.

The initial was a first-order "correction" approach that used correction terms to adjust the traditional FDTD update equations, thus simulating PML attenuation. Because the correction PML did not require any modification of the traditional FDTD update equations, it greatly reduced the programming complexity of PML implementation. The advantages of using a correction PML over traditional PML implementations were found to be as follows:

1) It increased the ease of PML implementation.

2) It could be added to existing codes without making any changes to the underlying update equations.

3) It offered seamless absorption in model corner regions without any special consideration.

4) It had identical absorption performance to traditional PML implementations.

5) It allowed for the implementation of arbitrary stretching functions, thus making the implementation of higher order PMLs straightforward.

6) Its recursive integration approach required less memory storage than alternative approaches, thus reducing computational requirements.

More importantly, in addition to the correction PML, a formulation to create higher order PMLs, of arbitrary order, was presented. To assess the ability of the new PML formulation to increase absorption, two examples were outlined. One example was an elongated rectangular full space and the other an elongated rectangular half-space. The key findings were as follows:

1) Second-order PMLs offer an increased number of degrees of freedom in comparison with first-order schemes, thus facilitating superior absorption performance.

2) Higher order PMLs require careful attenuation coefficient selection to maximize absorption and maintain PML stability.

3) Higher order PMLs require additional computational resources in comparison with first-order schemes. This additional computational cost must be carefully considered when selecting an appropriate PML formulation.

\section{ACKNOWLEDGMENTS}

The authors would like to acknowledge the facilities provided by the University of Edinburgh and Heriot-Watt University. Without these resources, this work would not have been possible.

\section{APPENDIX A}

\section{HIGHER ORDER PML'S FOR A GENERAL 3D CASE}

For a staggered scheme in which velocity components are updated at $t=n$ and stress components at $t=n+1 / 2$, the calculation of velocity for the entire grid is as follows:

$$
v_{i}^{n}=v_{i}^{n-1}+b \delta t\left(J_{i i}^{n}+J_{i j}^{n}+J_{i k}^{n}\right),
$$

where $\delta t$ is the time step size and $i, j, k \in[x, y, z]$. The PML velocity correction terms $J_{i i}, J_{i j}, J_{i k}$ are calculated using

$$
\begin{aligned}
J_{i j}^{n} & =\left\{\left(\prod_{q=1}^{N} R A_{j_{q}}\right)-1\right\} \frac{\partial d_{i j}^{n}}{\partial j} \\
& +\sum_{p=1}^{N-1}\left\{\left(\prod_{q=p+1}^{N} R A_{j_{q}}\right) R B_{j_{q}} \Phi_{i j}^{n-1}\right\}+R B_{j_{N}} \Phi_{i j_{N}}^{n-1},
\end{aligned}
$$

where $N$ is the order of the PML and $p \in[1, N]$. The previous time integral $\Phi_{i j_{p}}^{n}$ is obtained through

$$
\begin{aligned}
\Phi_{i j_{p}}^{n} & =R E_{j_{p}} \Phi_{i j_{p}}^{n-1}-R F_{j_{p}}\left\{\left(\prod_{q=1}^{p-1} R A_{j_{q}}\right) \frac{\partial d_{i j}^{n}}{\partial j}\right. \\
& \left.+\sum_{m=1}^{p-1}\left(\prod_{q=m+1}^{p-1} R A_{j_{q}}\right) R B_{j_{m}} \Phi_{i j_{m}}^{n-1}\right\} .
\end{aligned}
$$

Similarly, stresses at $t=n+1 / 2$ can then be calculated using

$$
d_{i j}^{n+1 / 2}=d_{i j}^{n+1 / 2}+\delta t C_{i j k l}\left(M_{i i}^{n+1 / 2}+M_{i j}^{n+1 / 2}+M_{i k}^{n+1 / 2}\right),
$$

with the correction terms found using

$$
\begin{aligned}
M_{i j}^{n+1 / 2} & =\left\{\left(\prod_{q=1}^{N} R A_{j_{q}}\right)-1\right\} \frac{\partial v_{j}^{n+1 / 2}}{\partial j} \\
& +\sum_{p=1}^{N-1}\left\{\left(\prod_{q=p+1}^{N} R A_{j_{q}}\right) R B_{j_{q}} \Phi_{i j}^{n-1 / 2}\right\}+R B_{j_{N}} \Phi_{i j_{N}}^{n-1 / 2},
\end{aligned}
$$

and the previous time integral

$$
\begin{aligned}
\Phi_{i j}^{n+1 / 2} & =R E_{j_{p}} \Phi_{i j_{p}}^{n-1 / 2}-R F_{j_{p}}\left\{\left(\prod_{q=1}^{p-1} R A_{j_{q}}\right) \frac{\partial v_{j}^{n+1 / 2}}{\partial j}\right. \\
& \left.+\sum_{m=1}^{p-1}\left(\prod_{q=m+1}^{p-1} R A_{j_{q}}\right) R B_{j_{m}} \Phi_{i j}^{n-1 / 2}\right\} .
\end{aligned}
$$


For all $J_{i j}^{n}$ and $M_{i j}^{n+1 / 2}$,

$$
\begin{aligned}
R A_{z_{p}} & =\frac{2+\Delta t \alpha_{z_{p}}}{2 \kappa_{z_{p}}+\Delta t\left(\alpha_{z_{p}} \kappa_{z_{p}}+d_{z_{p}}\right)}, \\
R B_{z_{p}} & =\frac{2 \kappa_{z_{p}}}{2 \kappa_{z_{p}}+\Delta t\left(\alpha_{z_{p}} \kappa_{z_{p}}+d_{z_{p}}\right)}, \\
R E_{z_{p}} & =\frac{2 \kappa_{z_{p}}-\Delta t\left(\alpha_{z_{p}} \kappa_{z_{p}}+d_{z_{p}}\right)}{2 \kappa_{z_{p}}+\Delta t\left(\alpha_{z_{p}} \kappa_{z_{p}}+d_{z_{p}}\right)}, \\
R F_{z_{p}} & =\frac{2 d_{z_{p}} \Delta t}{\left(2 \kappa_{z_{p}}+\Delta t\left(\alpha_{z_{p}} \kappa_{z_{p}}+d_{z_{p}}\right)\right) \kappa_{z_{p}}} .
\end{aligned}
$$

Beyond the PML region $d=0, \alpha=0, \kappa=1$, resulting in $R A=1, R B=1, R E=1$, and $R F=0$. This causes $J_{i i}, J_{i j}, J_{i k}$ to reduce to $\partial d_{i j}^{n} / \partial z$ and $M_{i i}, M_{i j}, M_{i k}$ to reduce to $\partial v_{j}^{n+1 / 2} / \partial z$. Therefore, stress and velocity equations automatically revert to the original stress derivatives.

\section{REFERENCES}

Abarbanel, S., and D. Gottlieb, 1997, A mathematical analysis of the PML method: Journal of Computational Physics, 134, 357-363, doi: 10.1006/ jcph.1997.5717.

Basu, U., 2009, Explicit finite element perfectly matched layer for transient three-dimensional elastic waves: International Journal for Numerical Methods in Engineering, 77, 151-176, doi: 10.1002/nme.

Basu, U., and A. K. Chopra, 2004, Perfectly matched layers for transient elastodynamics of unbounded domains: International Journal for Numerical Methods in Engineering, 59, 1039-1074, doi: 10.1002/nme .896.

Bécache, E., A. Ezziani, and P. Joly, 2003, Mathematical and numerical modeling of wave propagation in linear viscoelastic media, in G. C. Cohen, P. Joly, E. Heikkola, and P. Neittaanmäki, eds., Mathematical and numerical aspects of wave propagation: Springer, 916-921.

Berenger, J. P., 1994, A perfectly matched layer for the absorption of electromagnetic waves: Journal of Computational Physics, 114, 185-200, doi: 10.1006/jcph.1994.1159.

Berenger, J., 1996, A perfectly matched layer for free-space simulation in finite-difference computer codes: Annales Des Télécommunications, 51, 39-46, doi: 10.1007/BF02995690.

Cerjan, C., D. Kosloff, R. Kosloff, and M. Resheq, 1985, A nonreflecting boundary condition for discrete acoustic and elastic wave equations: Geophysics, 50, 705-708, doi: 10.1190/1.1441945.

Chew, W., and Q. Lui, 1996, Perfectly matched layers for elastodynamics: A new absorbing boundary condition: Journal of Computational Acoustics, 4, 341-359, doi: 10.1142/S0218396X96000118.

Chew, W., and W. Weedon, 1994, A 3D perfectly matched medium from modified Maxwell's equations with stretched coordinates: Microwave and Optical Technology Letters, 7, 599-604, doi: 10.1002/mop .4650071304 .

Collino, F., and P. Monk, 1998, Optimizing the perfectly matched layer: Computer Methods in Applied Mechanics and Engineering, 164, 157171, doi: 10.1016/S0045-7825(98)00052-8

Collino, F., and C. Tsogka, 2001, Application of the perfectly matched absorbing layer model to the linear elastodynamic problem in anisotropic heterogeneous media: Geophysics, 66, 294-307, doi: 10.1190/1.1444908.

Correia, D., and J. Jin, 2005, On the development of a higher-order PML: IEEE Transactions on Antennas and Propagation, 53, 4157-4163, doi: 10 1109/TAP.2005.859901.

Drossaert, F., and A. Giannopoulos, 2007, Complex frequency shifted convolution PML for FDTD modelling of elastic waves: Wave Motion, 44, 593-604, doi: 10.1016/j.wavemoti.2007.03.003.
Drossaert, F. H., and A. Giannopoulos, 2007, A nonsplit complex frequencyshifted PML based on recursive integration for FDTD modeling of elastic waves: Geophysics, 72, no. 2, T9-T17, doi: 10.1190/1.2424888.

Festa, G., and J. Vilotte, 2005, The Newmark scheme as velocity-stress timestaggering: An efficient PML implementation for spectral element simulations of elastodynamics: Geophysical Journal International, 161, 789812, doi: 10.1111/j.1365-246X.2005.02601.x.

Giannopoulos, A., 2012, Unsplit implementation of higher-order PMLs: IEEE Transactions on Antennas and Propagation, 60, 1479-1485, doi: 10.1109/TAP.2011.2180344.

Graves, R. W., 1996, Simulating seismic wave propagation in 3D elastic media using staggered-grid finite differences: Bulletin of the Seismological Society of America, 86, 1091-1106.

Guddati, M. N., and K. Lim, 2006, Continued fraction absorbing boundary conditions for convex polygonal domains: International Journal for Numerical Methods in Engineering, 66, 949-977, doi: 10.1002/nme .1574.

Hagstrom, T., and S. I. Hariharan, 1998, A formulation of asymptotic and exact boundary conditions using local operators: Applied Numerical Mathematics, 27, 403-416, doi: 10.1016/S0168-9274(98)00022-1.

Hastings, F., J. B. Schneider, and S. L. Broschat, 1996, Application of the perfectly matched layer (PML) absorbing boundary condition to elastic wave propagation: Journal of the Acoustical Society of America, 100, 3061-3069, doi: 10.1121/1.417118.

Higdon, R. L., 1986, Absorbing boundary conditions for difference approximations to the multi-dimensional wave equation: Mathematics of Computation, 47, 437-439, doi: 10.2307/2008166.

Komatitsch, D., and R. Martin, 2007, An unsplit convolutional perfectly matched layer improved at grazing incidence for the seismic wave equation: Geophysics, 72, no. 5, SM155-SM167, doi: 10.1190/1.2757586.

Kouroussis, G., L. Van Parys, C. Conti, and O. Verlinden, 2014, Using threedimensional finite element analysis in time domain to model railway-induced ground vibrations: Advances in Engineering Software, 70, 63-76, doi: 10.1016/j.advengsoft.2014.01.005.

Kouroussis, G., O. Verlinden, and C. Conti, 2011, Finite-dynamic model for infinite media: Corrected solution of viscous boundary efficiency: ASCE Journal of Engineering Mechanics, 137, 509-511, doi: 10.1061/(ASCE) EM.1943-7889.0000250.

Kuzuoglu, M., and R. Mittra, 1996, Frequency dependence of the constitutive parameters of causal perfectly matched anisotropic absorbers: IEEE Microwave and Guided Wave Letters, 6, 447-449, doi: 10.1109/75 .544545 .

Laghrouche, O., and D. Le Houédec, 1994, Soil-railway interaction for active isolation of traffic vibration, in M. Papadrakakis, and B. H. V. Topping, eds., Advances in simulation and interaction techniques: CivilComp Press, 31-36.

Lysmer, J., and R. Kuhlemeyer, 1969, Finite dynamic model for infinite media: Journal of Engineering Mechanics, 95, 859-878.

Martin, R., D. Komatitsch, and A. Ezziani, 2008, An unsplit convolution perfectly matched layer improved at grazing incidence for seismic wave propagation in poroelastic media: Geophysics, 73, no. 4, T51-T61, doi: 10.1190/1.2939484.

Martin, R., D. Komatitsch, S. D. Gedney, and E. Bruthiaux, 2010, A highorder time and space formulation of the unsplit perfectly matched layer for the seismic wave equation using auxiliary differential equations (ADEPML): Computer Modelling in Engineering and Sciences, 56, 17-42.

Peng, C., and M. Toksoz, 1995, An optimal absorbing boundary condition for elastic wave modeling: Geophysics, 60, 296-301, doi: 10.1190/1 1443758.

Roden, J. A., and S. D. Gedney, 2000, Convolutional PML (CPML): An efficient FDTD implementation of the CFS-PML for arbitrary media: Microwave and Optical Technology Letters, 27, 334-339, doi: 10.1002/ 1098-2760(20001205)27:5<334::AID-MOP14>3.0.CO;2-A.

Virieux, J., 1986, P-SV wave propagation in heterogenous media: Velocitystress finite-difference method: Geophysics, 51, 889-901, doi: 10.1190/1 1442147.

Zeng, C., J. Xia, J. Miller, and G. Tsoflias, 2011, Application of the multiaxial perfectly matched layer (M-PML) to near-surface seismic modeling with Rayleigh waves: Geophysics, 76, no. 3, T43-T52, doi: 10.1190/1 3560019 .

Zhang, W., and Y. Shen, 2010, Unsplit complex frequency-shifted PML implementation using auxiliary differential equations for seismic wave modeling: Geophysics, 75, no. 4, T141-T154, doi: 10.1190/1.3463431. 